\title{
Near-wake characteristics of rigid and membrane wings in ground effect
}

\author{
R. Bleischwitz ${ }^{1, *}$, R. de Kat ${ }^{2}$, B. Ganapathisubramani ${ }^{3}$ \\ Aerodynamics $\mathcal{E}$ Flight Mechanics Group \\ Faculty of Engineering and the Environment \\ University of Southampton \\ Southampton, SO17 1BJ, UK
}

\begin{abstract}
Wind tunnel measurements are conducted at a Reynolds numbers of $R e=56,000$ to examine the characteristics of near-wake vortices of rigid flat-plates and membrane wings from free-flight into ground-effect conditions. Synchronised high-speed load cell measurements, digital image correlation and particle image velocimetry are performed to resolve lift, drag and pitch oscillations simultaneously with membrane deformation and flow dynamics. Flow measurements are acquired in a crossflow-plane, one chord downstream of the trailing-edge, allowing the examination of time evolution of the wake and its relationship to the forces and membrane deformation. Membrane wings are found to delay ground-effect or high angles-of-attack induced tip-vortex break-down and result in larger tip-vortex push-out (beyond the wing span) compared to rigid flat-plate wings. The leading-edge vortex appears to shed with streamwise vorticity close to the root of the wing and this frequency of this shedding is found to match with the dominant frequency observed in membrane fluctuations. In specific resonance conditions, membrane and flow fluctuations are found to correlate well to the fluctuations in loads and moments.
\end{abstract}

Keywords: Membrane wings, fluid-structure interaction, ground-effect, Micro-Air-Vehicles, compliant wings, low Reynolds number wings

\footnotetext{
${ }^{*}$ Corresponding author

Email address: robert.bleischwitz@gmail.com (R. Bleischwitz)

${ }^{1}$ Post-doc

${ }^{2}$ Research Fellow

${ }^{3}$ Professor
} 


\section{Nomenclature}

$\begin{array}{llll}\alpha & \text { global angle-of-attack }\left[{ }^{\circ}\right] & L E / T E & \text { leading / trailing edge } \\ \Gamma & \text { Circulation }\left[\mathrm{m}^{2} / \mathrm{s}\right] & M & \text { membrane listener point } \\ \Pi & \text { aeroelastic parameter }\left(\frac{E t}{q c}\right)^{1 / 3} & P O D & \text { proper orthogonal decomposition } \\ \sigma & \text { standard deviation } & P S D & \text { power spectral density } \\ c & \text { chord length } & q & \text { dynamic pressure } \frac{1}{2} \rho U_{\infty}^{2} \\ C C F & \text { cross correlation function } & R e & \text { Reynolds number } \frac{U_{\infty} c}{{ }^{\circ}} \\ C_{D} & \text { drag coefficient } & S t & \text { Strouhal number } \frac{\frac{f c}{U_{\infty}}}{U_{\infty}} \\ C_{L} & \text { lift coefficient } & U & \text { streamwise flow velocity }[\mathrm{m} / \mathrm{s}] \\ h & \text { height above ground } & V & \text { vertical flow velocity }[\mathrm{m} / \mathrm{s}] \\ L & \text { flow listener point } & W & \text { spanwise flow velocity }[\mathrm{m} / \mathrm{s}]\end{array}$




\section{Introduction}

The recent interest in advanced Mirco-Air-Vehicles has lead into a series of studies to investigate biological inspired wings for low Reynolds number $\left(\mathrm{Re}=10^{4}-10^{5}\right.$ ) flow conditions (Bleischwitz et al., 2015b; Song et al., 2008; Rojratsirikul et al., 2010b). Their progress is challenged by a low energetic flow regime with a weak boundary layer, resulting in early flow separation, limitations in lift production and aerodynamic performance. The usage of bat-inspired thin and flexible membrane wings is found to be one possibility to improve low Reynolds number wing performance by enableling flow-induced static camber increase in combination with membrane oscillations (Rojratsirikul et al., 2011). The resulting fluid-structure coupling between the flexible membrane of the wing and passing-by vortical shedding structures is found to entrain energy into the flow, resulting in dynamic flow reattachment with enhanced lift production and delay of stall-angles (Zhang et al., 2016; Arce et al., 2013). However, the added lift production of flexible membrane wings comes at a price of higher drag, limiting their maximum aerodynamic efficiency (Bleischwitz et al., 2015b; Timpe et al., 2013; Gordnier and Attar, 2014).

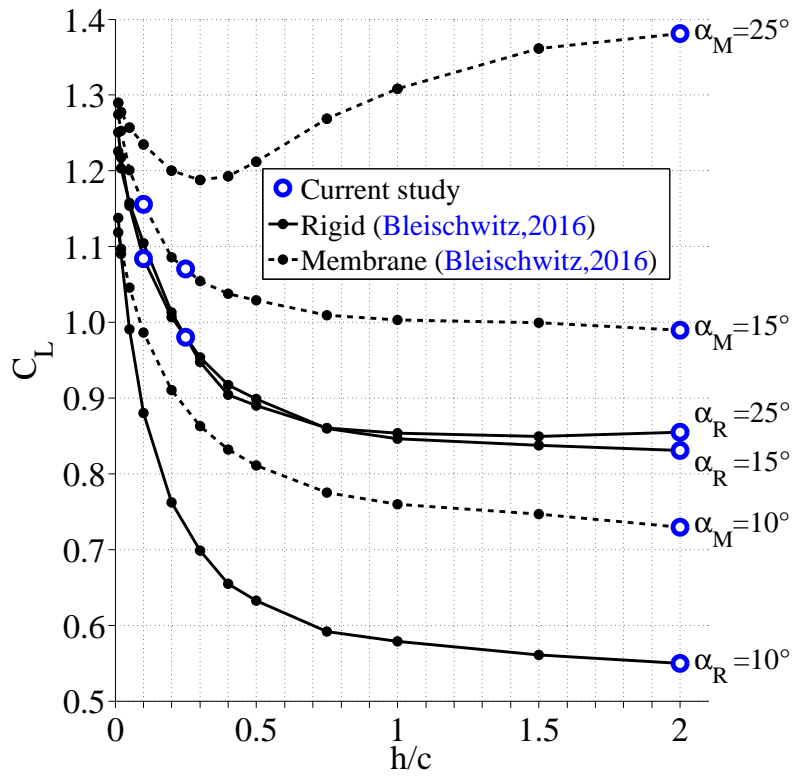

(a) Lift coefficient

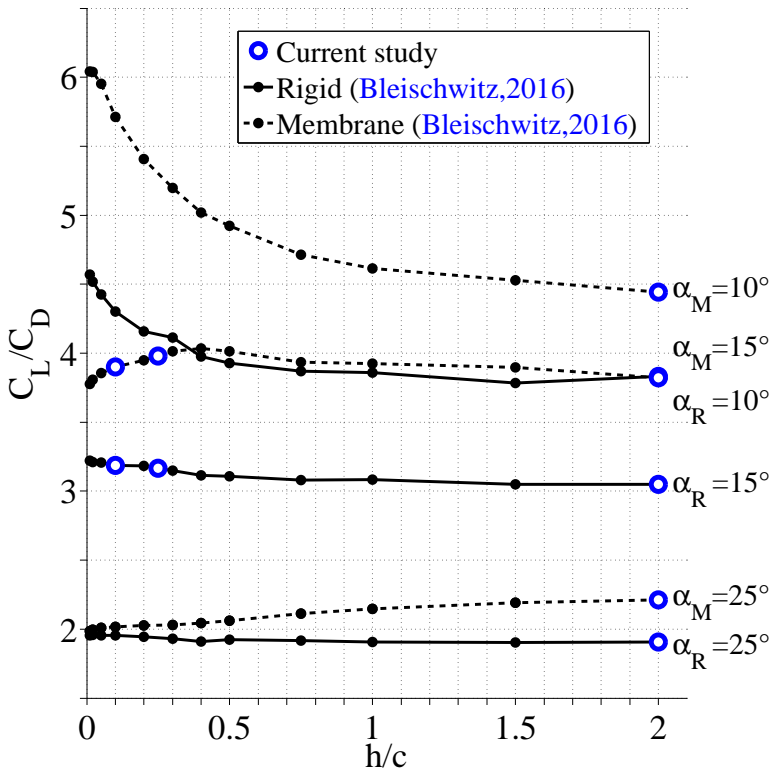

(b) Aerodynamic efficiency

Fig. 1 Background information extracted from the initial parameter study, comparing rigid flat-plates and membrane wings in and out-off ground-effect (Bleischwitz et al., 2016): (a) Static lift capability (b) and aerodynamic efficiency, going from free-flight (h/c $=2)$ into ground-effect conditions $(\mathrm{h} / \mathrm{c} \leq 1)$, measured at three angles-of-attack $\left(\alpha=[10,15,25]^{\circ}\right)$. Selected heights about ground and angles-of-attack of the present study are reflected with blue circles $\left(\alpha_{R}=\alpha_{\text {Rigid }}, \alpha_{M}=\alpha_{\text {Membrane }}\right)$.

To overcome low Reynolds number related efficiency limitations, flying in ground-effect could be one option to expand the overall aerodynamic performance. A wing is said to be in ground-effect (GE), if its height from the trailing-edge to the ground is either lower than half of the wingspan or below one chord length (Yun et al., 2010). Performance improvements result from a stagnation of the flow below the wings surface, causing a static pressure increase on the lower side of the wing (Vogt and Barber, 2012). As a result, more flow is not only diverted over the upper wing surface but also pushed out along the span direction, extending virtually the aspect-ratio of wings in ground-effect (Han and Cho, 2005). Consequently, the induced drag component can be reduced and leads ultimately to gains in aerodynamic efficiency of wings in ground-effect conditions (Ahmed and Sharma, 2005; Ahmed et al., 2006).

By combining flexible membrane wings and ground-effect, one might expect to tackle both stall as well as performance related issues. Several studies on fish-related propulsion, using flexible panels in close-to-ground proximity, seems to back-up the given hypothesis by showing flexibility related performance gains close to the ground (Quinn 
et al., 2014; Park et al., 2017). In addition, recent wind tunnel experiments at the University of Southampton investigated both static wing performance as well as coupling effects of rigid and flexible membrane wings in and out of ground-effect (Bleischwitz et al., 2015a, 2016, 2017). Time-sychnronised high-speed measurements were recorded, including dynamic loads, wing deformations and streamwise flow modifications. Fig. 1 shows lift (Fig. 1a) and aerodynamic performance (Fig. 1b) of rigid flat-plates and membrane wings, going from free-flight $(\mathrm{h} / \mathrm{c}=2)$ into ground effect conditions $(\mathrm{h} / \mathrm{c} \leq 1)$. Three angles-of-attack at $\alpha=[10,15,25]^{\circ}$ are shown for brevity. At typical free-flight conditions $(\mathrm{h} / \mathrm{c}=2)$, both rigid flat-plates and membrane wings show higher lift production with inclining angle-of-attack (Fig. 1a). The gain in lift should be accompanied by modifications to the wing-tip vortices. These wing-tip modifications could have an influence on downstream bodies that are in its wake. Unfortunately, wing-tip modifications were not examined in the previous study (Bleischwitz et al., 2016, 2017).

One prior experimental study on wing-tip vortices of membrane wings was carried out by Rojratsirikul et al. (2010b) who measured wing-tip vortices of rigid flat-plates and membrane wings at free-flight conditions and concluded that membrane wings exhibit stronger tip vortices compared to rigid flat-plate wings. Similarly, Gordnier and Attar (2014) investigated tip-vortex modifications computationally and distinguish additionally between rigid flat-plates, rigid cambered and membrane wings. This important study shows that the growth in wing mean-camber results in an increase in tip-vortex strength over all angles-of-attack. In addition, membrane oscillations are found to impact tipvortex modifications specifically at higher angles-of-attack. With increasing angle-of-attack, tip-vortices were found to relocate towards the wing root and membrane wing surface, accompanied with a gain in vortex size and strength. Both above-mentioned tip-vortex related membrane wing studies were conducted in free-flight conditions (without ground-effect) and examined mainly flow statistics. In this study, we examine the structure of wing-tip vortices of rigid and membrane wings and relate that to simultaneously measured membrane deformations and load dynamics.

Wind tunnel experiments are conducted at $R e=56,000$, measuring time-resolved load (load-cell), membrane (digital image correlation, DIC) and flow (particle image velocimetry, PIV) quantities for membrane wings at a selection of angles-of-attack of $\alpha=[10,15,25]^{\circ}$ in free-flight $(\mathrm{h} / \mathrm{c}=2)$ and at $\alpha=15^{\circ}$ in ground-effect conditions $(\mathrm{h} / \mathrm{c}=[0.25$, 0.1]). Rigid flat-plates are used for comparison to be consistent with our previous studies (Bleischwitz et al., 2016, 2017). Flow measurements are conducted in a cross-flow plane, located one chord length downstream of the trailingedge in the wake. The selected PIV-plane enables us to study tip-vortex modifications as well as any vortex-shedding patterns in the wake. Time-averaged flow results are used to extract ground-effect induced tip-vortex relocation along the span and vertical direction. Time-resolved flow recordings in the wake allow us to identify dominant vortical flow structures and their spectral content. Flow quantities are filtered via proper orthogonal decomposition (POD) and correlated to membrane and load dynamics for understanding underlying relationship between tip-vortex signature, membrane deformation and loads. Overall, this work is a continuation of our previous work reported in Bleischwitz et al. (2017). The previous work focused on flowfield on top of the wing while this current study addresses the tipvortex and the wake. 


\section{Experimental Setup}

Measurements were conducted in an open-loop, low-speed blow-down wind tunnel at the University of Southampton. The test section measures $48 \mathrm{~cm}$ width by $43 \mathrm{~cm}$ height with a free-stream turbulence intensity of less than $0.1 \%$. The turbulence level was measured with hot-wire anemometry. The wind speed could be varied between 2 to $14 \mathrm{~m} / \mathrm{s}$ and was fixed to $8.4 \mathrm{~m} / \mathrm{s}$, reaching a Reynolds number of $R e=56,000$.

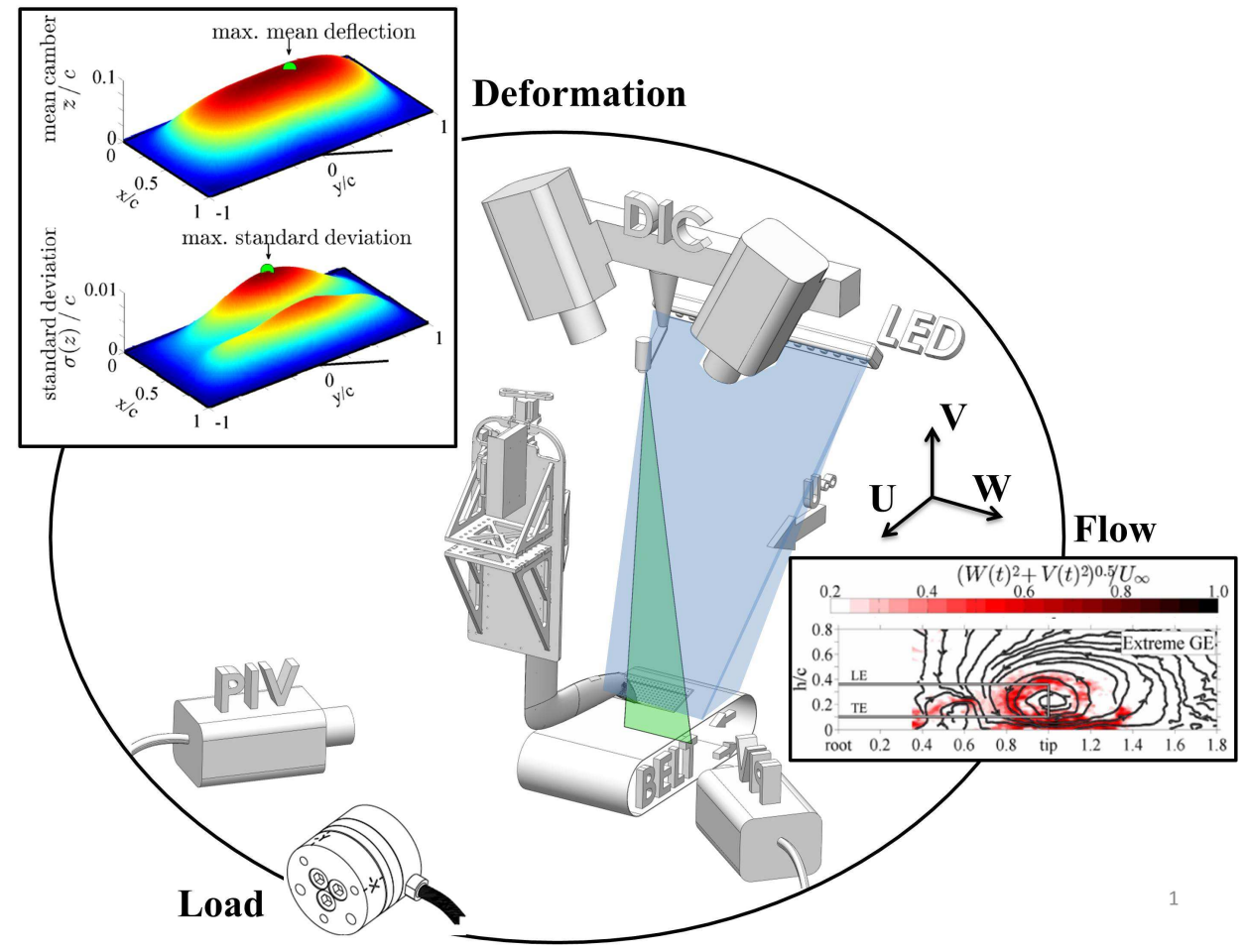

Fig. 2 Experimental setup with time-sychnronised load (sting integrated load-cell), deformation (stereo Digital-ImageCorrelation) and wake related flow (stereo Particle-Image-Velocimetry) measurements one chord downstream of the trailing-edge. The LED illumination for the membrane motions is illustrated with blue shading, whereas the freestream-normal, flow-related laser-plane is colored in green.

The current experimental setup equipment (Fig. 2) is similar to those used in our previous work (Bleischwitz et al., 2016, 2017) and focuses on time-synchronised and high-speed load, membrane and flow (wake) measurements in the cross-flow plane. Key elements of the setup consist of a robotic L-shaped sting-arm for pitch and height control of the wings and a rolling-road system for reflecting ground-effect conditions. Further details about the design and development of all experimental equipment can be found in the PhD-Thesis (Bleischwitz, 2016). 


\subsection{Wing Design}

The experiment uses full-wing models with a rectangular planform shape, measuring $100 \mathrm{~mm}$ in chord and 200 $\mathrm{mm}$ in span and reflecting an aspect-ratio of $\mathrm{AR}=2$ (Fig. 3). The rigid flat-plate consists of a $1 \mathrm{~mm}$ transparent acyclic plate which is surrounded by a round, $3 \mathrm{~mm}$ diameter perimeter steel frame. The latex sheet material has a thickness of $\mathrm{t}=0.2 \mathrm{~mm}$, density of $1 \mathrm{~g} / \mathrm{cm}^{3}$ and a stiffness of $\mathrm{E}=1.5 \mathrm{MPa}$. The aeroelastic parameter $\Pi=(\mathrm{Et} / \mathrm{qc})^{1 / 3}$ (Smith and Shyy, 1996) is found to be $\Pi=4.27$ for $U_{\infty}=8.4 \mathrm{~m} \cdot \mathrm{s}^{-1}$. Further design details of the wing construction can be found in Bleischwitz et al. (2017).

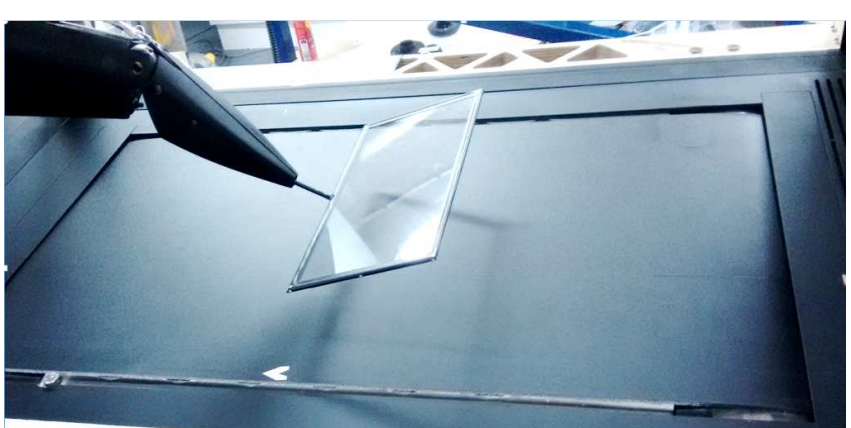

(a) Sting mounted wings with rolling-road

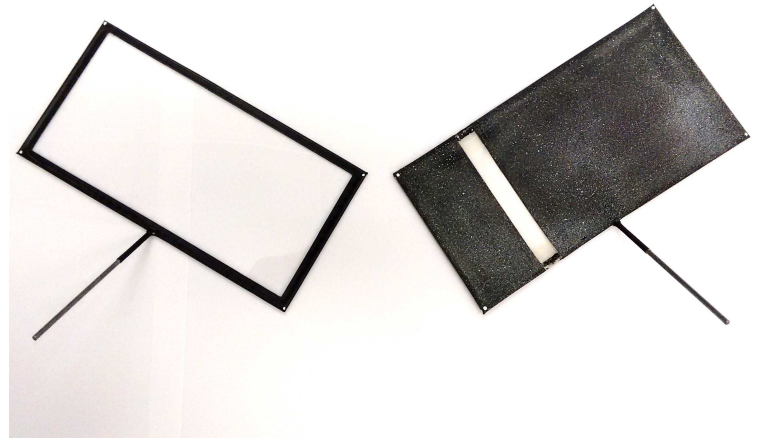

(b) Rigid flat-plate and membrane wing

Fig. 3 Wing design and setup integration

It is worth noting that the specific $A R=2$ is chosen based on the results of our previous study with that focused on aspect-ratio effects (Bleischwitz et al., 2015b), which showed that lower aspect-ratio membrane wings $(A R<2)$ would delay the onset of leading-edge vortex-shedding and would restrict coupling of the flow with the membrane at preferable low spatial frequency membrane modes. In addition, key literature on membrane wings with tip-vortex effects use a comparable $\mathrm{AR}=2$, chord length and aeroelastic parameter which showed the ability of lag-free fluidmembrane coupling (Rojratsirikul et al., 2010a; Gordnier and Attar, 2014).

\subsection{Load Measurement}

Forces and moments are measured by a six-axis load cell (ATI-Nano 17, load capacity $25 \mathrm{~N}$ ) which is integrated into the sting system. A National Instrument (NI USB-6343, 16-bit) data acquisition system is used to acquire the loads at a frequency of $10 \mathrm{kHz}$ and a time-period of $20 \mathrm{~s}$. The loads are further low-pass-filtered to $800 \mathrm{~Hz}$, allowing to correlate with the DIC and PIV related recording frequency. The uncertainty in the load cell is $\leq 0.006 \mathrm{~N}$ in forces and $\leq 0.03 \mathrm{Nmm}$ in pitching moment (ATI calibration, ISO 9001 certified).

The dynamic behavior of the experimental setup is separately validated by identifying the dynamics of setuprelated key-components (sting, fan, wing-frame). Fig. 4 illustrates exemplary lift based (load-cell acquired) PSDspectra of the setup without any wing (blue-dotted line), a flat-plate (red-line) and a membrane wing (black-line) installed. All machinery with the wind tunnel were switched on. The wing-related case (free-flight at $\alpha=25^{\circ}$ ) involves stall-conditions with strong flow-induced excitation energy for the membrane. The eigen frequencies of the wing frame (made of steel rods) are identified with an impulse response-test as $19 \mathrm{~Hz}$ for the flat-plate and $25 \mathrm{~Hz}$ for the membrane wing (again, related to the steel frame). Their excited energy level measures $-40 \mathrm{~dB}$ to $-35 \mathrm{~dB}$. The sting eigen frequency was also identified before by a separate impulse-test and was found to be $33 \mathrm{~Hz}$ with a response of $-35 \mathrm{~dB}$. The wind tunnel blower-fan was found to be an important noise producer and could be measured as $-30 \mathrm{~dB}$ at $53 \mathrm{~Hz}$. As a result, the shedding frequencies of rigid flat-plate wings were often dominated by the fan-noise and could not be resolved with the given load-cell (fortunately, they can be identified with the flow measurements). In comparison, the load-dynamics of membrane wings are strong enough and dominant load-dynamics were resolved at 


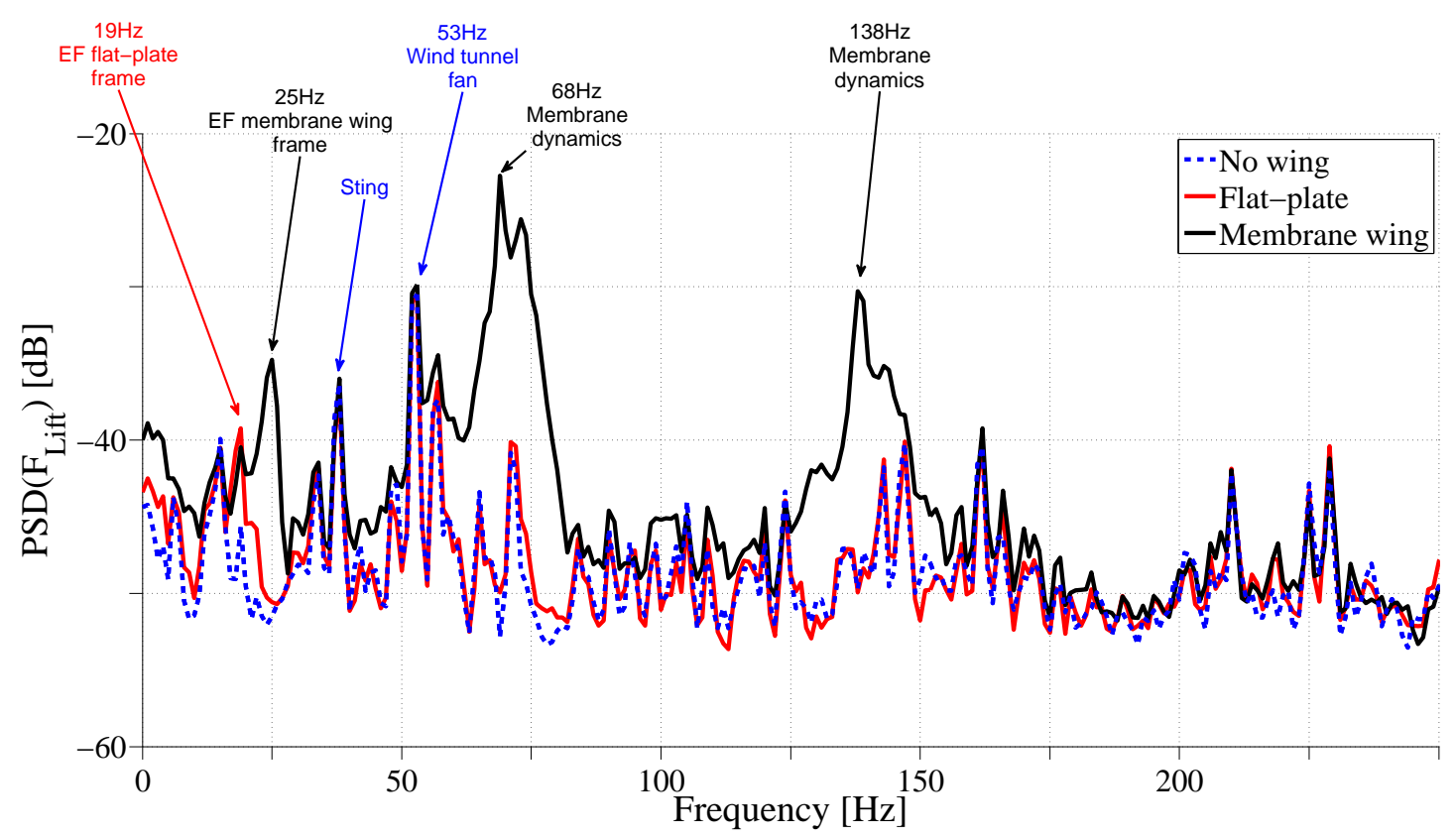

Fig. 4 Excited resonance behaviour of experimental setup with wind tunnel and systems switched on, recorded with load-cell. Cases: No wing mounted, flat-plate mounted, membrane wing mounted (free-flight at $\alpha=25^{\circ}$ ).

$68 \mathrm{~Hz}$ and $138 \mathrm{~Hz}$, showing generally higher energy levels of $\geq-30 \mathrm{~dB}$. The time-synchronised setup even allowed us to use spectral information of membrane and flow dynamics to trace specific unclear load frequencies to their origin.

For further details of the load-cell validation, the reader is referred to the complete doctoral thesis (Bleischwitz, 2016).

\subsection{Deformation Measurement}

Stereoscopic digital image correlation (DIC) is carried out to measure instantaneous membrane deformations using two high-speed cameras (Phantom V341) with $50 \mathrm{~mm}$ focal length lenses (Nikon AF Nikkor 50mm f/1.8D) and an aperture set to $\mathrm{f} / 8$. The DIC cameras were mounted with a $60^{\circ}$ included angle between each other, which stays fixed for a given height-over-ground and modifications in angle-of-attack. As a result, every height-over-ground position had a separate calibration of the measurement volume. An aluminium calibration plate with dot-pattern is used to image at six different angle-of-attack positions (covering the angle-of-attack range). The volume reconstruction (for a given height) is conducted using a commercial software LaVision StrainMaster.

The black membrane is sprayed with a white random speckle pattern with $\sim 3$ to 5 speckles per mm $(\sim 2$ pixels per speckle). The patterns are illuminated with blue light-emitting-diodes. To avoid interference of the (green) PIVlaser-light during simultaneous DIC and PIV recordings, the DIC camera-lenses are equipped with blue bandpass filters. High-speed images are captured at a sampling-rate of $800 \mathrm{~Hz}$ over a sampling-time of $6.25 \mathrm{~s}$. The sampling frequency allows resolving dynamics in the membrane below $400 \mathrm{~Hz}$. A total of 5000 images per camera are obtained at single-frame. LaVision StrainMaster is used to obtain deformations from the stereo images. The uncertainty of the membrane deformation is estimated to be no worse than $0.1 \%$ of the chord length and is measured by recording two images in still position while comparing the displacement between them. The given uncertainty represents worst-case conditions. The DIC-technique is widely used in experimental mechanics, allowing sub-pixel accuracy due to grey value interpolation schemes over the interrogation grid (Schreier et al., 2000). The uncertainty values and overall membrane deformations agree with previous DIC-setups and measurements on comparable membrane wings (Rojratsirikul et al., 2011; Galvao et al., 2006; Stanford et al., 2014).

Fig. 2 shows an example of time-averaged out-of-plane membrane surface deformations and fluctuations. It is worth to mention that vortex-shedding induced membrane vibrations are typically in a range of $10 \%$ of the mean 
membrane deflection ( 0.5 to $2 \mathrm{~mm}$ in our case).

\subsection{Flow Measurement}

Stereoscopic flow measurements (stereo particle-image-velocimetry) are conducted in a cross-flow plane, located one chord length downstream of the trailing-edge. Fig. 2 shows the instantaneous in-plane kinetic energy contours $\left(\sqrt{\left(W^{2}+V^{2}\right)} / U_{\infty}\right)$ and streamline patterns. The downstream position of the stereo-PIV-plane is selected to capture a fully developed tip-vortex structure of sufficient size and allows to track its modification with changes in angleof-attack and height-over-ground. Two Phantom V341 cameras (same type as used for DIC) are arranged in a stereo configuration and are both aligned $45^{\circ}$ to the laser plane, resulting in a $90^{\circ}$ included angle between the two PIV cameras. Each camera is additionally tilted with respect to the lens axis using Scheimpflug-adapters and is focused at the centre of the PIV-measurement plane. The cameras are equipped with a set of two $105 \mathrm{~mm}$ focal length lenses (Sigma $105 \mathrm{~mm} \mathrm{f} / 2.8 \mathrm{EX}$ DG Macro) with aperture set to f/2.8 (fully open). Green bandpass filters are added on the PIV-lenses to increase the signal-to-noise ratio for the cameras by filtering out the background light from the (DICrelated) LEDs. A double-cavity Nd: YLF laser (Litron LDY-300) with a wavelength of $527 \mathrm{~nm}$ is used. The flow is seeded with $1 \mu \mathrm{m}$ droplets generated using glycerine-water mixture in a typical atomiser. The image-pair acquisition frequency of $800 \mathrm{~Hz}$ allows a resolution of $1616 \times 1088$ pixels for each camera, resulting in a field-of-view of $17 \mathrm{~cm} \times$ $8 \mathrm{~cm}$ (span $\times$ height). A total of 5000 double-frame images are recorded over a time of $6.25 \mathrm{~s}$. The time delay between image-pairs is set to $\Delta t=30 \mu \mathrm{s}$, resulting in a peak particle image displacement of about 4 pixels. The imagespairs are mapped by using the information of a two-level calibration plate and processed into vector fields. The final interrogation window size was $32 \times 32$ pixels with $75 \%$ overlap, which results in an in-plane spatial resolution of 0.43 vectors $/ \mathrm{mm}$. The commercial software LaVision Flowmaster ${ }^{T M}$ is used for image acquisition and post-processing of particle images into vector fields.

Measurements were conducted at a selection of angles-of-attack of $\alpha=[10,15,25]^{\circ}$ at $\mathrm{h} / \mathrm{c}=2$ and $\alpha=15^{\circ}$ for $\mathrm{h} / \mathrm{c}=[0.25,0.1]$, where the height-over-ground $(h)$ is referenced with respect to the trailing-edge of the wing. The specifically chosen cases (of base study Bleischwitz et al. (2016)) allow to show the transition from attached flow conditions to flow separation with (fluid-membrane coupling related) dynamic flow reattachment.

\subsection{Synchronisation}

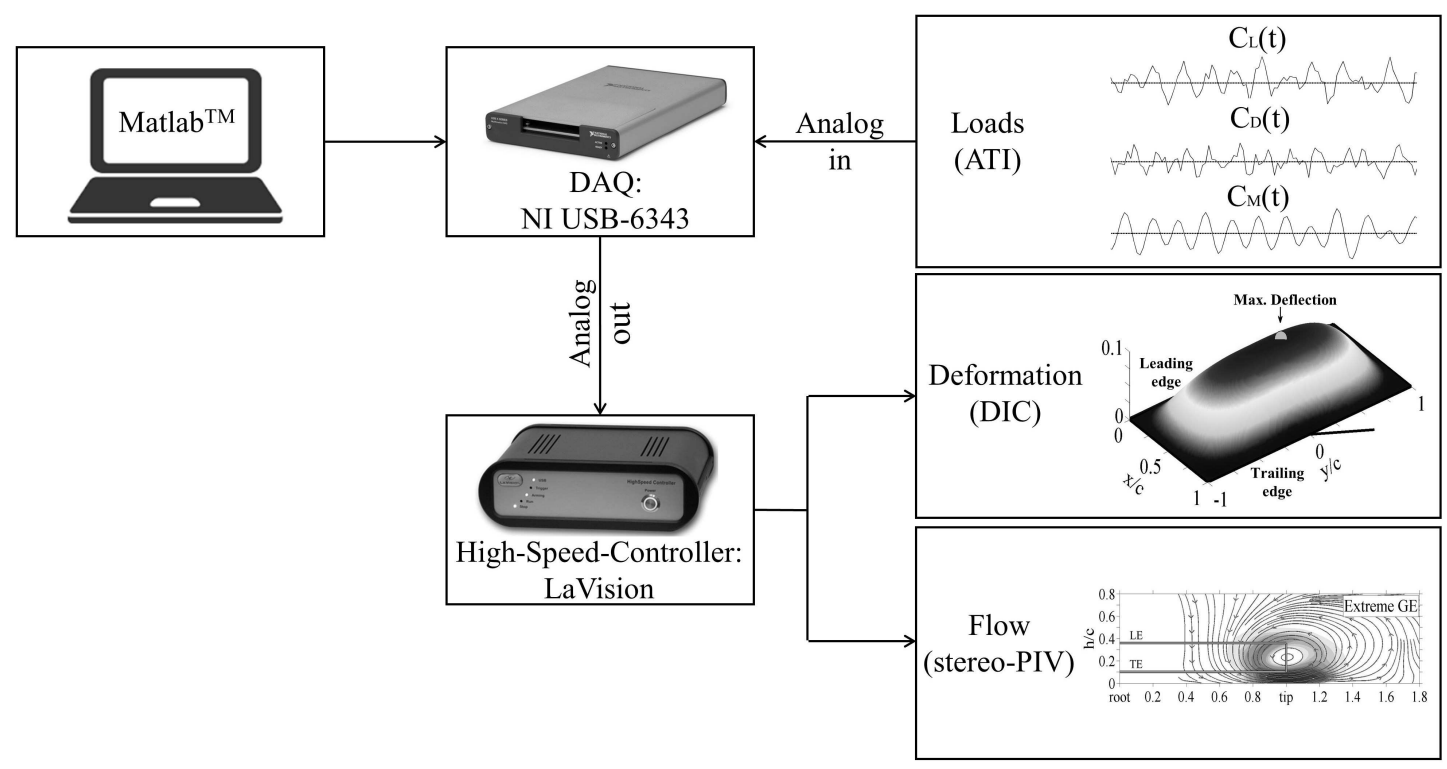

Fig. 5 Synchronisation layout of combined load, deformation and flow measurement 
The time-synchronisation between the two deformation cameras (DIC), the two flow measurement cameras (stereoPIV) and the laser is ensured with the help of a LaVision high-speed controller. The high-speed controller itself is triggered via a analog output-signal from a data-acquisition-system (DAQ, National-Instrument USB-6343). In parallel, the DAQ ensures to record the analog input channels of the ATI-load-cell in parallel. MATLAB ${ }^{T M}$ is used to control the DAQ. Fig. 5 illustrates the synchronisation layout for clarity. The time-synchronisation over all systems was practically tested by fixing the wing at $45^{\circ}$ roll-angle, which enabled all cameras (DIC + PIV) to view at the same time the wing surface. Than, an object ( 5 gram) was released above the wing to fell via gravity onto the wings metal framing. The image number (timing), where the object hits the frame and start to deform the wing-frame, is compared for all four cameras (for testing in single-frame) and checked with the timing of the rising load-impulse.

\section{Results}

\subsection{Statistics}

Fig. 6 shows the effect of changes in angle-of-attack on the time-averaged in-plane velocity magnitude of the tipvortex $\left(\sqrt{\left(W^{2}+V^{2}\right)} / U_{\infty}\right)$. The in-plane velocity components $W$ (horizontal) and $V$ (vertical) are added as streamline patterns. Rigid flat-plates (Fig. 6a) and membrane wings (Fig. 6b) are compared. The perimeter wing frame is shown projected into the given cross-stream PIV-plane (only half-span is visible), allowing to reference the tip-vortex size and its center-core position in relation to the wing frame. For completeness, the (time-averaged) location of the tipvortex center-core is calculated from the peak axial vorticity and is summarised for all height- and angle-of-attack cases in Fig. 10a.
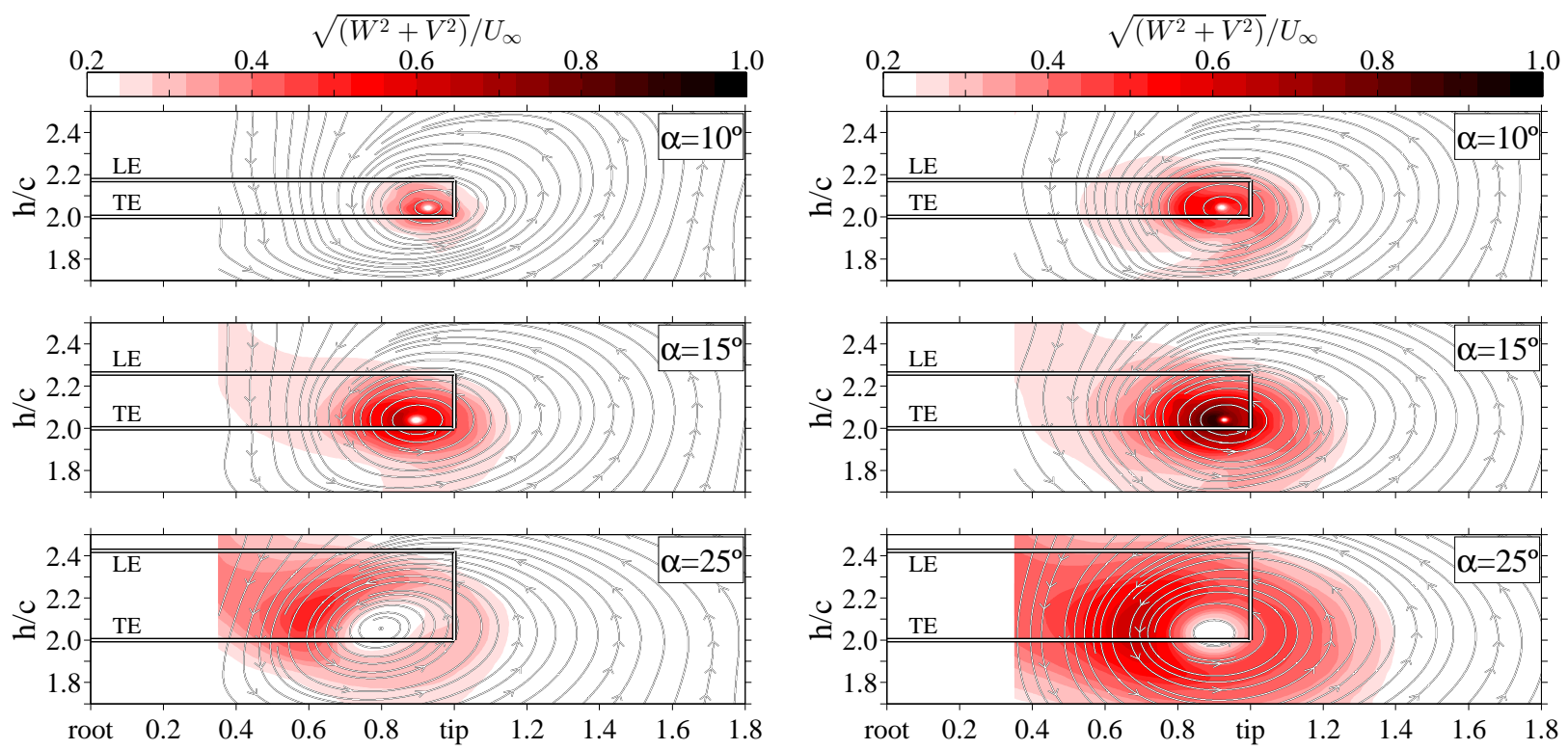

(a) Rigid flat-plate

(b) Membrane

Fig. 6 Effect of angle-of-attack on tip-vortex velocity magnitude and its position to the projected wing frame. Comparison of rigid flat-plate and membrane wings at $\alpha=[10,15,25]^{\circ}$ in free-flight conditions $(h / c=2)$.

Both rigid flat-plate (Fig. 6a) and membrane wings (Fig. 6b) show an increase in size of wing-tip vortex with increasing angle-of-attack of $\alpha=[10,15,25]^{\circ}$. This is primarily related to increase in lift with increasing angle-ofattack (see prior Fig. 1a).

In addition, with increasing angle-of-attack, both rigid flat-plate and membrane wings show that the centre of the tip-vortex moves inwards along the span towards the wing-root (see Fig. 6 and the extracted locations in Fig. 10a, top). This is consistent with previous studies at similar free-flight conditions (Gordnier and Attar, 2014). 


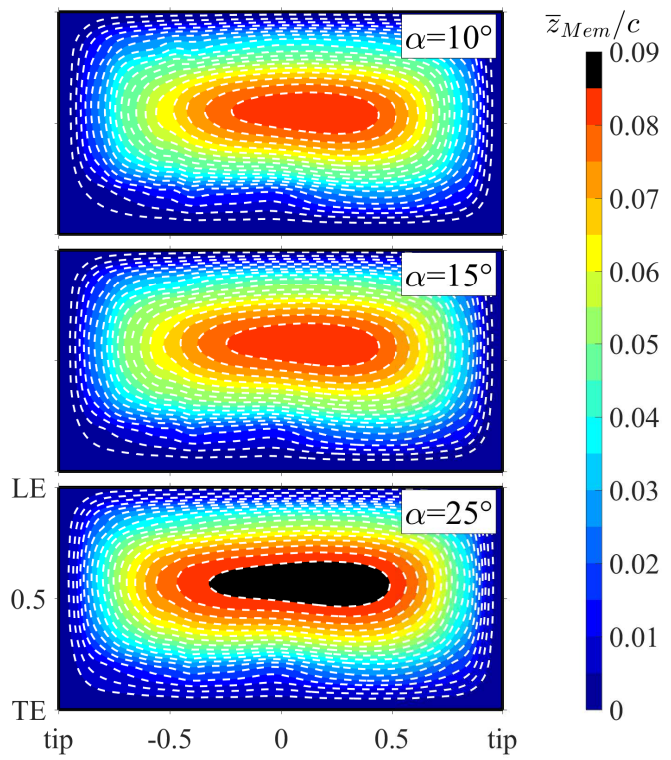

(a) Mean Deformation

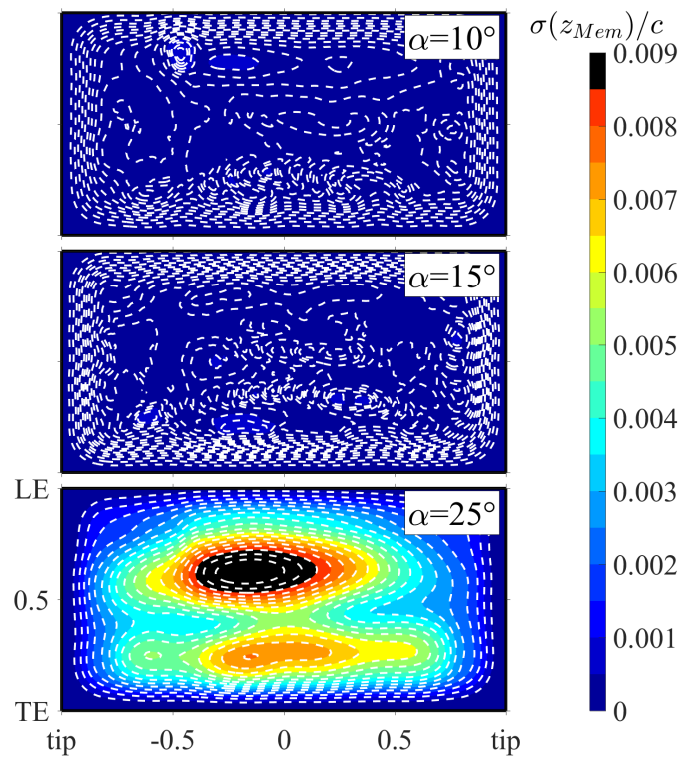

(b) Standard Deviation

Fig. 7 Effect of angle-of-attack $\alpha=[10,15,25]^{\circ}$ on membrane wing mean deflection $\left(\bar{z}_{M e m} / c\right)$ and standard deviation $\left(\sigma\left(z_{M e m}\right) / c\right)$. Membrane wing in free-flight conditions $(h / c=2)$.

Membrane wings (Fig. 6b) show generally stronger tip-vortices to rigid flat-plate wings (Fig. 6a), which is consistent with their higher lift production (Fig. 1a) due to flow-induced static membrane cambering and membrane oscillations. Fig. 7 shows the impact of an increased angle-of-attack on membrane mean deflections (Fig. 7a) and time-averaged membrane dynamics (Fig. 7b). Prior related studies have shown (Bleischwitz et al., 2017) that membrane wings can suppress leading-edge vortex-shedding up to an angle-of-attack of $\alpha=15^{\circ}$. As a result, membrane wings at lower angles-of-attack exhibit predominantly static membrane billowing (Fig. 7a) without significant membrane dynamics (Fig. 7b). At very high angles-of-attack of $\alpha=25^{\circ}$, membrane wings shows strong leading-edgeshedding induced membrane mode-shape excitation with two peaks (Fig. 7b, lower). The relation between membrane and wake-flow dynamics will be discussed at a later stage in this paper.

Fig. 8 shows the effect of changes in height-over-ground $(h / c=[2,0.25,0.1])$ on the time-averaged in-plane velocity magnitude $\left(\sqrt{\left(W^{2}+V^{2}\right)} / U_{\infty}\right)$. A specific angle-of-attack of $\alpha=15^{\circ}$ was chosen based on previous results presented in Bleischwitz et al. (2017), reflecting the transition region from flow conditions at free-flight to groundeffect conditions. Both rigid flat-plate and membrane wings show with their descent into ground-effect significant horizontal and vertical translations of the tip-vortex core compared to the projected wing frame. Membrane wings show a horizontal push-out of their tip-vortex center of up to $30 \%$, ranging from $0.9 c$ in free-flight to $1.2 c$ in extreme GE conditions (Fig. 8b and Fig. 10a lower). In contrast, rigid flat-plate wings show a smaller ground-effect initiated horizontal push-out of up to $11 \%$ (Fig. 8a and Fig. 10a lower). Differences in the horizontal tip-vortex push-out might be linked with membrane wings ability to camber (Fig. 9a) and oscillate (Fig. 9b, lower), allowing to delay ground-effect initiated flow separation on the upper wing surface (shown prior in(Bleischwitz et al., 2017)). As a result, membrane wings are found to delay the tip-vortex break-down and allow a virtually expended aspect-ratio in comparison to rigid flat-plate wings.

ground-effect does not only affect horizontal, but also the vertical location of the tip-vortex (Fig. 10a lower). The vertical location of the tip-vortex is driven by the interaction with the solid boundary of the ground, forcing flow structures to be directed away from the surface. Both rigid flat-plate and membrane wings exhibit similar vertical tip-vortex locations of $\Delta h / c=[0.06$ to 0.07] (with reference to the wing-frame), while going from free-flight $(h / c=$ 2 ) into ground-effect conditions $(h / c=0.1)$. Tip-vortex locations and modifications along the streamwise direction 

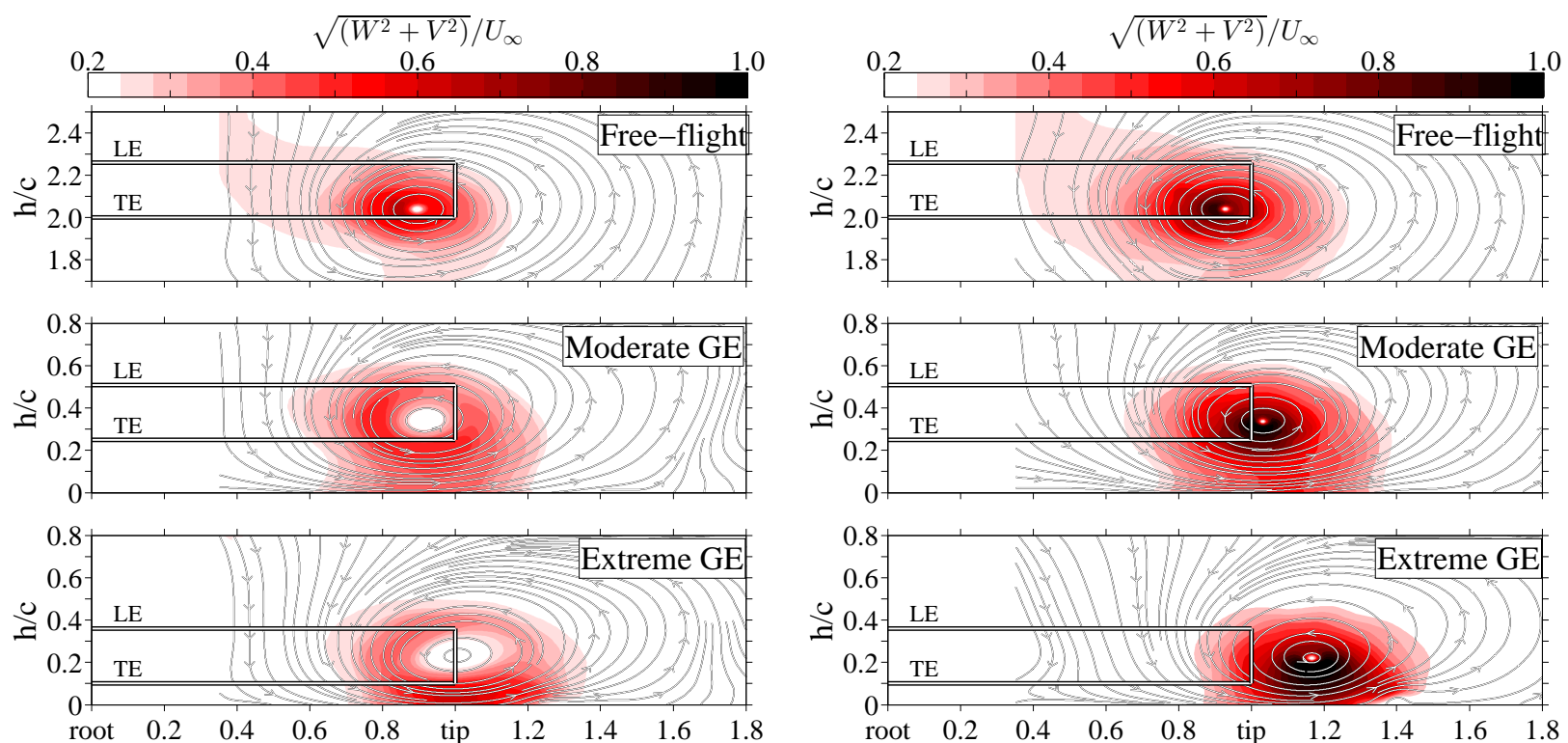

(a) Rigid flat-plate

(b) Membrane

Fig. 8 Effect of height-over-ground on tip-vortex velocity magnitude and its position to the projected wing frame. Comparison of rigid flat-plate and membrane wings at $\alpha=15^{\circ}$.

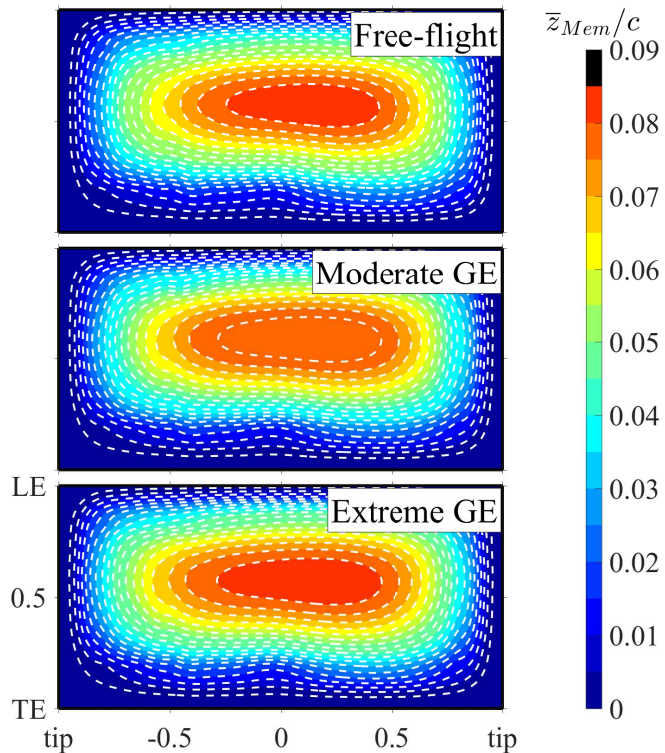

(a) Mean Deformation

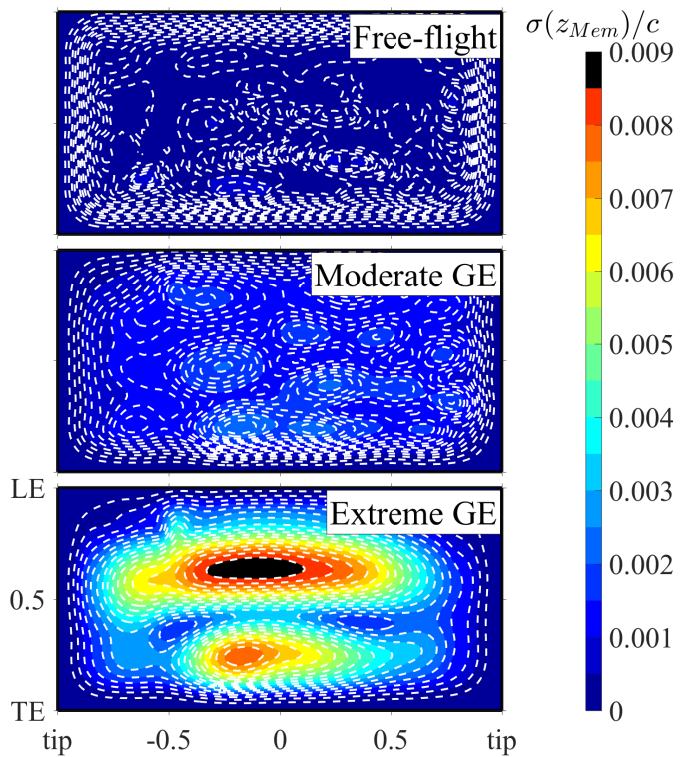

(b) Standard Deviation

Fig. 9 Effect of height-over-ground on membrane wing mean deflection $\left(\bar{z}_{M e m} / c\right)$ and standard deviation $\left(\sigma\left(z_{M e m}\right) / c\right)$. Membrane wing at $\alpha=15^{\circ}$. 
remain unknown, as the this study relates on a single PIV-plane, located one chord downstream of the trailing-edge.

Fig. 10b shows the magnitude of normalised circulation $\left(\Gamma / U_{\infty} c\right)$ of the tip-vortex for rigid flat-plate and membrane wings for various angles-of-attack (Fig. 10b, top) and heights-over-ground (Fig. 10b, bottom). The circulation was calculated from the area integral of vorticity distribution. The area integral is reflected by the projected, combined field-view of both PIV cameras, measuring $150 \mathrm{~mm}$ x $80 \mathrm{~mm}$ (span x height). All heights relate to the same integral area size, given from the projected PIV-field. The circulation of both rigid flat-plates and membrane wings is compared to a similar study of Rojratsirikul et al. (2010b), showing a good match for both wings in free-flight conditions (Fig. 10b, top). Ground-effect (Fig. 10b, bottom) is found to affect the circulation much less than changes in angles-of-attack. This might be explained by the influence of two counteracting phenomena. First, the descent into ground-effect results in higher lift production, which behaves similar to an increase in angle-of-attack (Fig. 1a). As a result, the circulation at the wing tip should increase and reflect a higher pressure difference at the wing-tip. However, ground-effect is also known to constrain the development of the tip-vortex size and strength due to spanwise flow diversion close to the ground (Han and Cho, 2005). Both effects seems to counter-balance each other, resulting in a relatively constant tip-vortex circulation values with varying height-over-ground (Fig. 10b, bottom).

It should be mentioned that similar circulation values for both rigid and membrane wings at extreme ground-effect $(h / c=0.1)$ might be a reflection of the fact that the predominant lift generation of both rigid and membrane wings is from the lower side of the wing. Both these cases show similar separated flow conditions on the upper wing surface (see prior study with streamwise PIV data by Bleischwitz et al. (2017)). As a result, membrane wings seem to behave similar to rigid flat-plates, as soon they are close to the ground and forced into extreme unsteady flow separation from the leading-edge. Further investigations are required to understand this similarity in more detail.
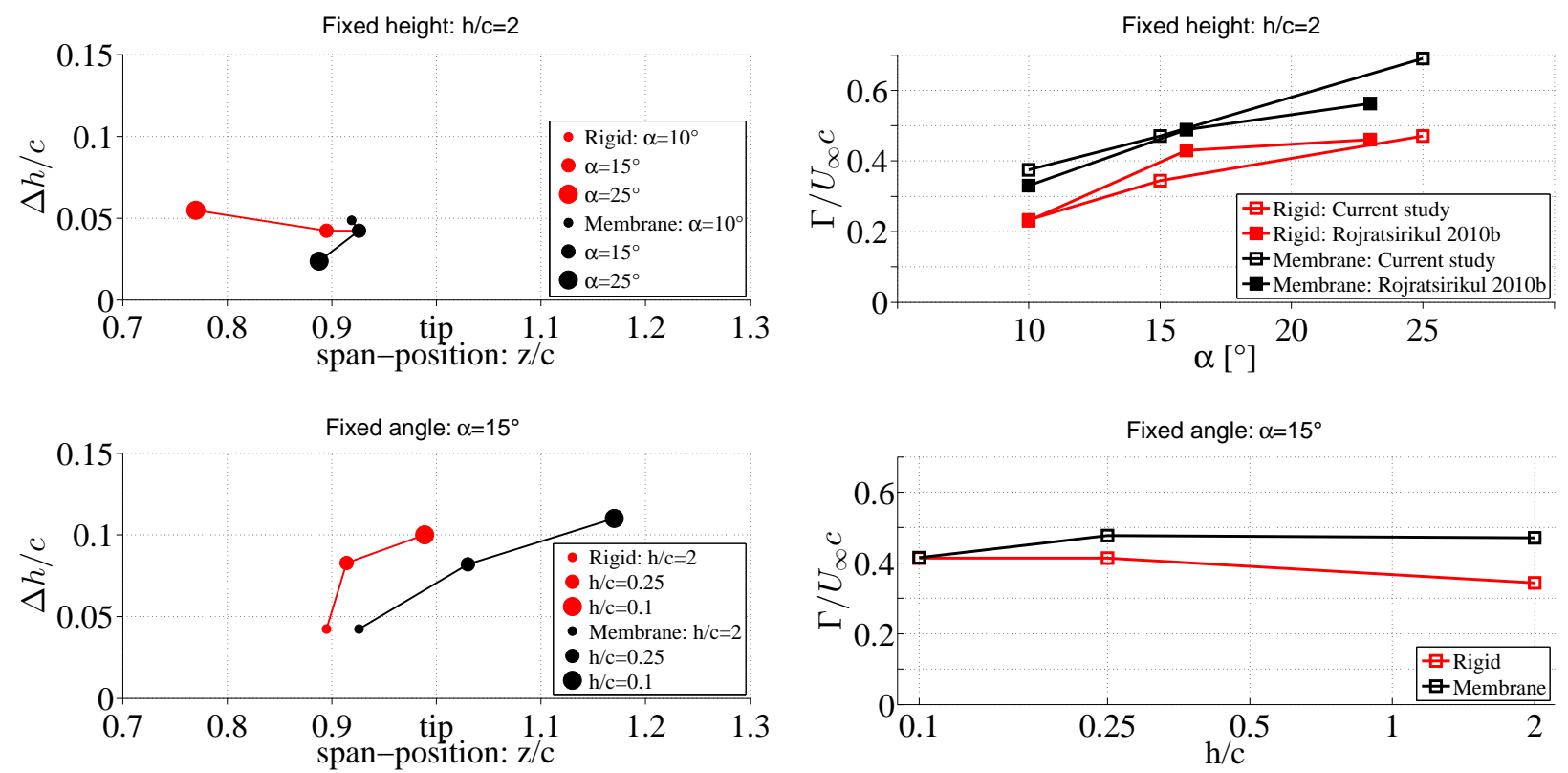

(a) Position of tip-vortex core $\left(h_{T V C}\right)$ relative to trailing-edge $\left(h_{T E}\right)$ $\left(\Delta h=h_{T V C}-h_{T E}\right)$ and wingspan. Modification in angle-of-attack at free-flight (upper plot) and changes in height-over-ground at $\alpha=15^{\circ}$ (lower plot).

(b) Normalized tip-vortex circulation for rigid flat-plate and membrane wings as function of angle-of-attack (upper plot) and height-over-ground (lower plot).

Fig. 10 Impact of ground-effect and angle-of-attack on (time-averaged) tip-vortex location and circulation. Comparison of rigid flat-plate and membrane wings.

\subsection{Wake Structures}

High-speed flow measurements allow to gain deeper insight in to underlying spatio-temporal evolution of flow structures. Fig. 11a shows an example of an instantaneous snapshot of freestream-normalised velocity fluctuations in 
cross-stream $\left(W^{\prime}\right.$ - horizontal, $V^{\prime}$ - vertical) and streamwise $\left(U^{\prime}\right)$ directions. The illustrated flow represents a membrane wing at $\alpha=15^{\circ}$ in extreme GE conditions $(h / c=0.1)$. The in-plane velocities $\left(W^{\prime}\right)$ and $\left(V^{\prime}\right)$ are also illustrated as streamline patterns. The diversity of apparent flow-scales and structures makes it often difficult to observe dominant vortical structures in instantaneous snapshots. Therefore, the velocity field is filtered using Proper Orthogonal Decomposition by retaining only the energetic modes. Almost all the large-scale structure is contained within the first 5 modes (beyond which the energy contained within an individual mode is less than $1 \%$ of the total energy). This POD-based filter is applied to each velocity component independently to ensure that the filter is not biased towards any specific velocity component. Note that this POD-based reconstruction is performed only to filter the data and not to obtain any specific flow physics. For implementation of the POD snapshot method, the reader is referred to previous work (Lumley, 1967; Sirovich, 1987; Pinnau, 2008).
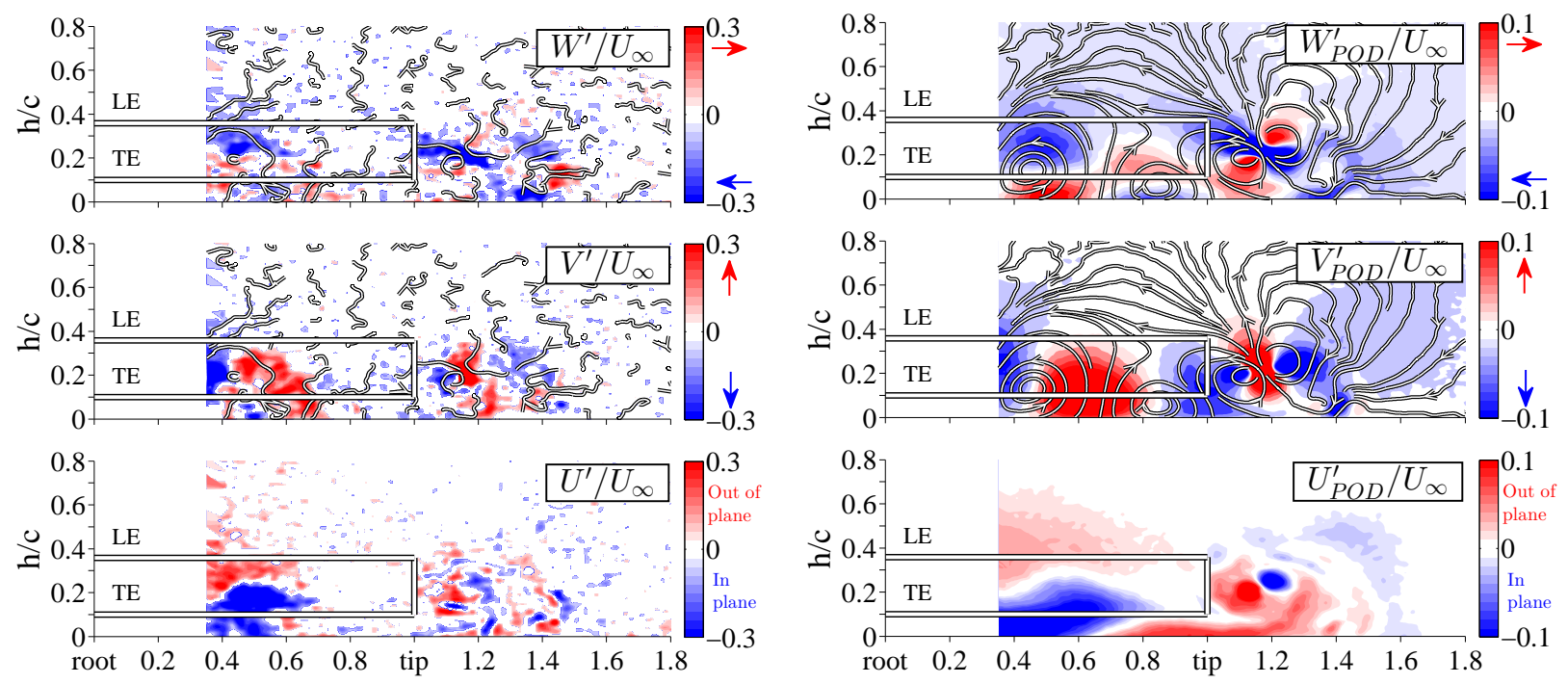

(a) Real flow

(b) POD reconstructed flow (from 5 POD-modes)

Fig. 11 (a) Instantaneous snapshot of freestream-normalised velocity fluctuations $U^{\prime}$ (out-of-plane), $V^{\prime}$ (vertical), $W^{\prime}$ (spanwise). Additionally, streamlines are illustrated, based on in-plane velocity fluctuation components $\left(V^{\prime}, W^{\prime}\right)$. (b) Effect of POD as filter technique on highly dynamic flow components and streamlines (using first five POD-modes).Membrane wing case at $\alpha=15^{\circ}$ in extreme GE $(h / c=0.1)$ conditions.

Fig. 11b shows the POD-snapshot reconstruction based on the first five most energetic POD-mode shapes. This filtered flowfield allows us to observe an important flow feature that occurs $0.4-0.8 c$ away from the root. The streamlines show a large vortex structure passing through the plane. The out-of-plane velocity component $\left(U_{P O D}^{\prime}\right.$, Fig. $11 \mathrm{~b}$, lower) reveals the 3-dimensional nature of the vortex, with flow speeding up at $h / c=0.4$ (red) and slowing down at $h / c=0.1$ (blue). This vortex structure can be interpreted as the signature of the leading-edge vortex that has shed and passed through the cross-stream PIV-plane in the wake. The result is consistent with our previous study that used only streamwise-wall-normal PIV measurements (Bleischwitz et al., 2017).

Fig. 12 illustrates the timeseries of Fig. 11 for a period of $\sim 15 \mathrm{~ms}$, which is equivalent to the time-scale of one shedding cycle of the leading-edge vortex. Unfiltered vertical flow oscillations (Fig. 12a) are compared with PODfiltered fluctuations (Fig. 12b). Again, the region around $0.4-0.8 c$ in span is of major interest, revealing a full cycle of vertical up-down flow dynamics, reflecting one LE-vortex shedding cycle which subsequently passes through the PIV wake-plane. For further visualisation of Fig. 12, supplementary videos are provided as Video-Fig.12a and VideoFig.12b.

High-speed stereo-PIV measurements in liaison with frozen flowfield hypothesis allows us to reconstruct the downstream spatial structure of the wake vortices. The convecting streamwise distance, $\Delta x$ can be computed assuming $\Delta x=U_{\infty} \Delta t$, where $U_{\infty}=8.4 \mathrm{~m} / \mathrm{s}$ and $\Delta t=\frac{1}{f_{P I V}}=\frac{1}{800 \mathrm{~Hz}}=1.25 \mathrm{~ms}$ ). Fig. 13 shows reconstructed vortical 


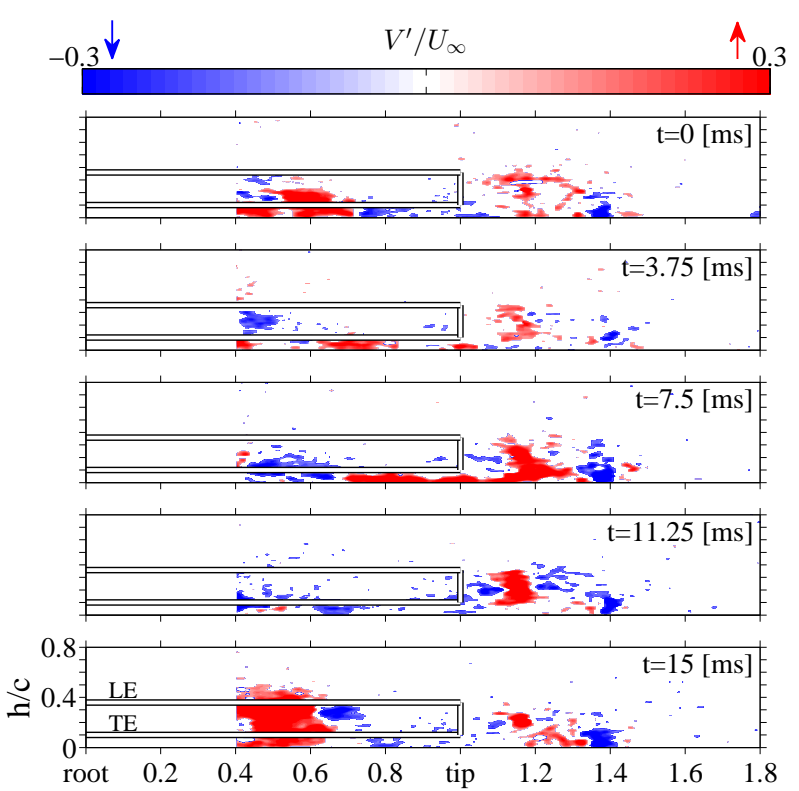

(a) Real-flow, Video-Fig.12a
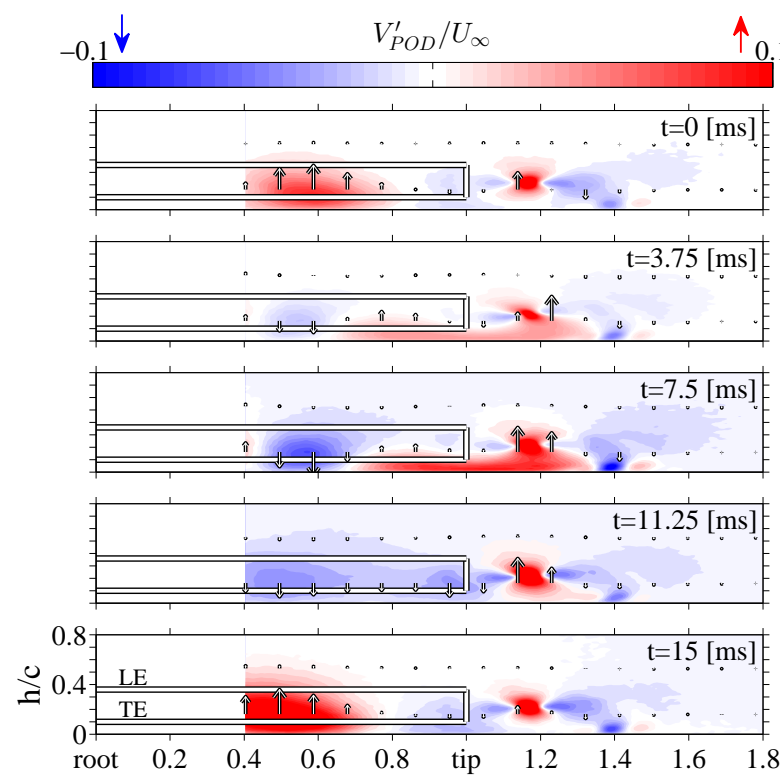

(b) POD reconstruction, Video-Fig. $12 \mathrm{~b}$

Fig. 12 (a) Timeseries of vertical flow fluctuations $V^{\prime}(t)$ (red=vertical up, blue=vertical down) and (b) filtered $V^{\prime}(t)$ that retain only the first 5 POD-modes. The flow cycle shows vertical flow oscillations between wing root and tip (0.4-0.8c in span). Membrane wing case at $\alpha=15^{\circ}$ in extreme GE $(h / c=0.1)$ conditions. (For illustration of the videos, the reader is referred to the web version of this paper.)

structures visualised using iso-surfaces of the Q-criterion (Dubief and Delcayre, 2000) which are color-coded with vertical velocity fluctuations (red $=$ upwards, blue $=$ downwards). The color-coding is consistent to our previous work (Bleischwitz et al., 2017), which revealed good correlation between vertical flow and vertical membrane dynamics. For clarity, flow structures are mirrored about the wing root (assuming symmetry).

Rigid flat-plate (Fig. 13, left) and membrane wings (Fig. 13, right) are compared at $\alpha=15^{\circ}$, going from free-flight $(h / c=2)$ into ground-effect conditions $(h / c=0.25,0.1)$. In free-flight, rigid flat-plate wings (Fig. 13a) show small and coherent tip-vortex structures as well as a defined vortex shedding structures close to the wing root, resulting in peak-lift conditions (Fig. 1a). The descent of rigid flat-plates into ground-effect (Fig. 13c,e) causes tip-vortex structures to break down and spread out in size. Membrane wings in free-flight conditions (Fig. 13b) show small and coherent tip-vortices with negligible vorticity closer to the root, reflecting attached flow conditions on the upper wing surface (see also streamwise PIV measurements in Bleischwitz et al. (2017)). Membrane wings are found to cope well with the adverse pressure gradient in ground-effect by showing consistency in tip-vortex size and their structural coherence (Fig. 13d,f). Nevertheless, membrane wings in ground-effect are also accompanied by growing patterns of vortex shedding close to the wing root. These results are consistent to our previous findings based on streamwise-wallnormal PIV measurements. For further visualisation, all subfigures of Fig. 13 are available as supplementary videos as Video-Fig.13a to Video-Fig.13f. 


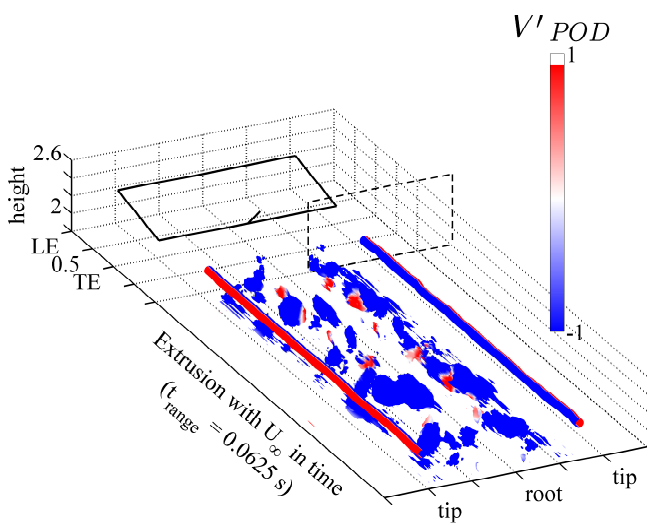

(a) Rigid flat-plate, $\mathrm{h} / \mathrm{c}=2$, Video-Fig.13a

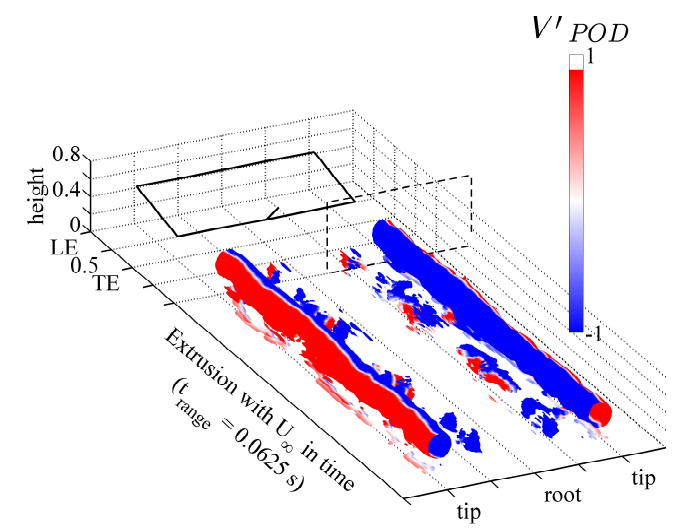

(c) Rigid flat-plate, $\mathrm{h} / \mathrm{c}=0.25$, Video-Fig. $13 \mathrm{c}$

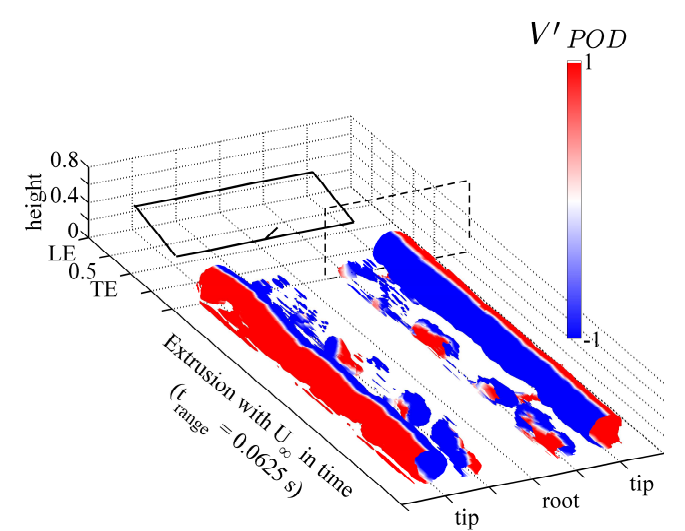

(e) Rigid flat-plate, $\mathrm{h} / \mathrm{c}=0.1$, Video-Fig.13e

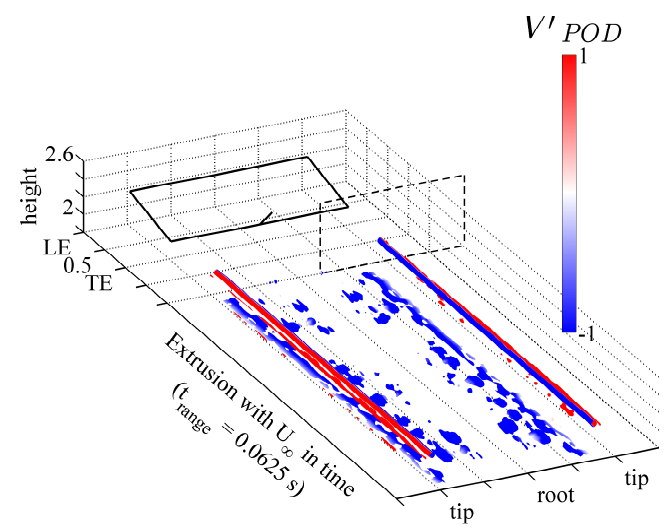

(b) Membrane, $\mathrm{h} / \mathrm{c}=2$, Video-Fig. $13 \mathrm{~b}$

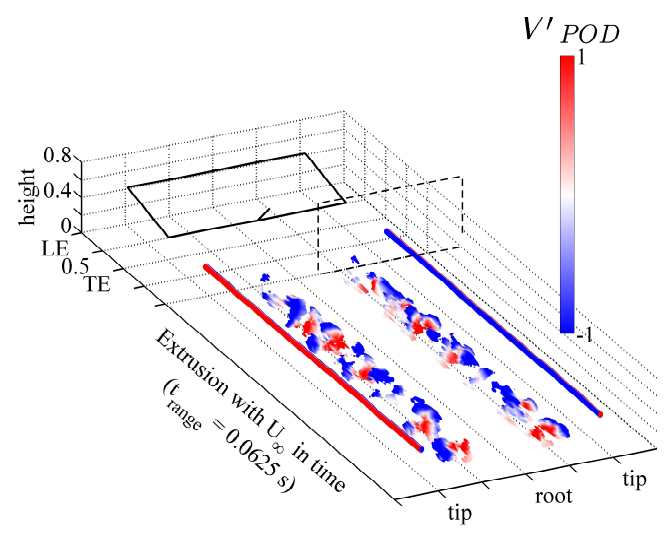

(d) Membrane, $\mathrm{h} / \mathrm{c}=0.25$, Video-Fig. $13 \mathrm{~d}$

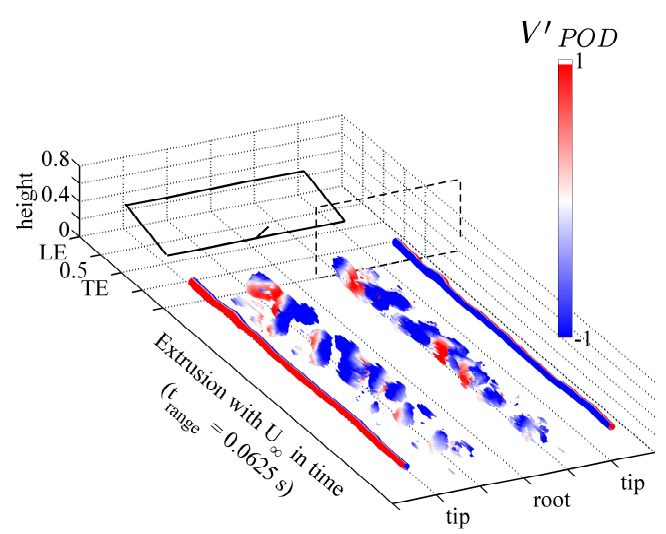

(f) Membrane, h/c = 0.1, Video-Fig. $13 \mathrm{f}$

Fig. 13 Freestream propagated vortical shedding structures, calculated via the iso-surfaces of the Q-criterion. The red/blue color coding represents (POD-filtered) vertical velocity fluctuations ( $r e d=$ upwards, blue $=$ downwards). Rigid flat-plate and membrane wings at $\alpha=15^{\circ}$ and $h / c=[0.1,0.25,2]$. (For illustration of Video-Fig.13a to Video-Fig.13f, the reader is referred to the papers web version.) 


\subsection{Spectral Content}

Fig. 14 shows the energy spectra of vertical flow oscillations $\left(P S D\left(V^{\prime}\right)\right)$ at five listener points along the span (points $L 1, \ldots, L 5$ are located in level with the trailing-edge). Vertical flow dynamics are chosen as they are clearer in showing correlation to the membrane dynamics (compared to horizontal flow fluctuations that have more broadband information). The listener points are illustrated within the contour plots of standard deviation of vertical velocity fluctuations $\sigma(V)$. The flow frequency is normalised by the chord length $c$ and free-stream velocity $U_{\infty}$ and is depicted as a Strouhal-number $S t=\frac{f c}{U_{\infty}}$. Rigid flat-plate and membrane wings are compared at $\alpha=25^{\circ}$ in free-flight $(h / c=2$, see Fig. 14a,b) and for $\alpha=15^{\circ}$ at moderate $(h / c=0.25$, see Fig. 14c,d) and in extreme ground-effect conditions $(h / c=0.1$, see Fig. 14e,f). In addition, all membrane wing cases (Fig. 14b,d,f) are shown with their spectral content in membrane oscillations (green line, based on membrane surface point with highest standard-deviation). For further details of the membrane dynamics, the reader is kindly referred to our previous study Bleischwitz et al. (2016). The illustrated (angle-of-attack and height) cases are specifically selected, as they show defined fluid-membrane coupling behavior, which were known from prior work (Bleischwitz et al., 2016, 2017).

The flow-based spectra reveals predominant frequencies close to the wing root (at listener $L 1$ ), which likely originates from leading-edge vortex-shedding. The shedding-frequencies are often difficult to capture, as the wake flow may contains a wide range of other flow-scales, known from previous studies (Gordnier, 2009; Gordnier and Attar, 2014). In addition, a growing strength and relocation of the tip-vortex may contribute to a broader scale distribution within the wake-flow plane, covering predominant leading-edge vortex-shedding frequencies with a diversity of other scales. As a result of a stronger tip-vortex influence, specific flow listener-points located close to the wing-tip appear broader in their flow spectra. Nevertheless, rigid flat-plate wings are found with a dominant shedding-frequency of $\mathrm{St}=0.54$ (Fig. 14a) and $\mathrm{St}=0.64$ (Fig. 14c,e) at listener point $L 1$ that agree well with the observations of previous studies on flat/cambered plates (Rojratsirikul et al., 2011). At this prior study, the Strouhal number was found to be in the range $0.17 \leq S t_{\text {wake }} \sin \alpha \leq 0.23$ (current study: $S t_{\text {wake }} \sin \alpha=0.22$ for Fig. $14 \mathrm{a}$ and $S t_{\text {wake }} \sin \alpha=0.17$ for Fig. 14c,e). Additionally, this flow frequencies match well with our prior results, measuring the similar wings within a streamwise PIV-plane (Bleischwitz et al., 2017).

The flow frequencies of membrane wings are found to modify and couple for specific cases with dominant vibration frequencies of the membrane (green line in Fig. 14b,d). Such membrane related flow frequencies appear generally higher than dominant flow frequencies seen for flat-plate wings. This finding is consistent with previous studies (Rojratsirikul et al., 2011; Timpe et al., 2013; Miranda et al., 2005). The first membrane wing case at $\alpha=15^{\circ}$ and $h / c=$ 0.1 (Fig. 14f) shows a membrane driven dominant shedding-frequency of $\mathrm{St}=0.74$ in the wake, which compares to a lower $\mathrm{St}=0.64$ frequency of rigid flat-plate wings (Fig. 14e). This specific membrane case in deep ground-effect is known to benefit from strong leading-edge vortex-shedding (Bleischwitz et al., 2017), combined with a ground-effect induced push-out of the tip-vortex, resulting in a well-defined coupling between membrane and shedding-related flow dynamics.

The second membrane wings case reflects more moderate ground-effect conditions of $\alpha=15^{\circ}$ and $h / c=0.25$ (Fig. 14d), where the energy entertainment of leading-edge vortex-shedding is not yet sufficient to allow synchronized coupling with the membrane dynamics. This can be seen with a shedding frequency of the flow of St $=1.37$, compared to a lower dominant membrane frequency of $\mathrm{St}=0.98$. This transient condition of membrane wing coupling is of importance, as it shows the transfer between uncoupled to coupled conditions. To trigger lag-free fluid-membrane coupling, a reduced height or increased angle-of-attack have to be engaged to excite well-defined resonance conditions.

The third membrane wing case (Fig. 14b) represents a coupled case at free-flight conditions $\left(\alpha=25^{\circ}\right.$ and $\left.h / c=2\right)$, where the dominant membrane frequency of $\mathrm{St}=0.83$ tries to couple and synchronize with the dominant leading-edge vortex-shedding frequency. However, strong tip-vortex entrainment results in a broadening effect of the wake spectra with large range of apparent flow scales. Ultimately, resonance conditions of fluid-membrane coupling can either be triggered by a reduction of height-over-ground or an increase in angle-of-attack. However, ground-effect seems to allow more distinct coupling conditions between the membrane and leading-edge vortex-shedding, as the tip-vortex influence can be pushed-out and suppressed. A recently published study of Zhang et al. (2017) uses comparable membrane wings to the current study, however with a (tip-vortex free) 2D-layout instead of the 3D-wing layout (which includes the effect of tip vortices) presented here. Their dominant shedding-frequencies appear more distinct compared to our current study, underlining the strong impact of the tip-vortex on the flow-scales within the wake. 

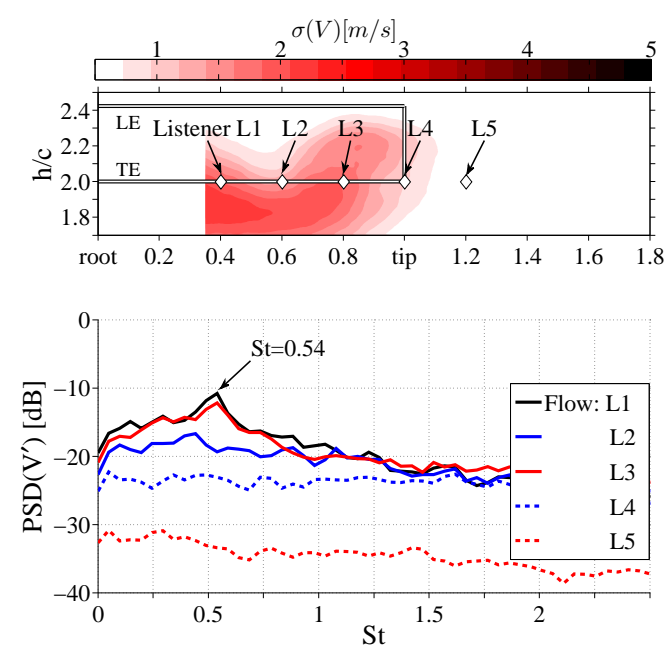

(a) Rigid flat-plate, $\mathrm{h} / \mathrm{c}=2, \alpha=25^{\circ}$
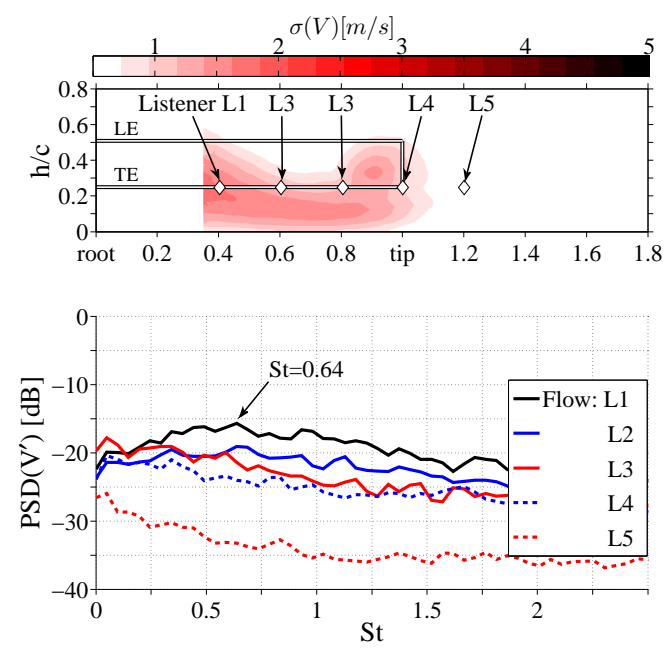

(c) Rigid flat-plate, $\mathrm{h} / \mathrm{c}=0.25, \alpha=15^{\circ}$
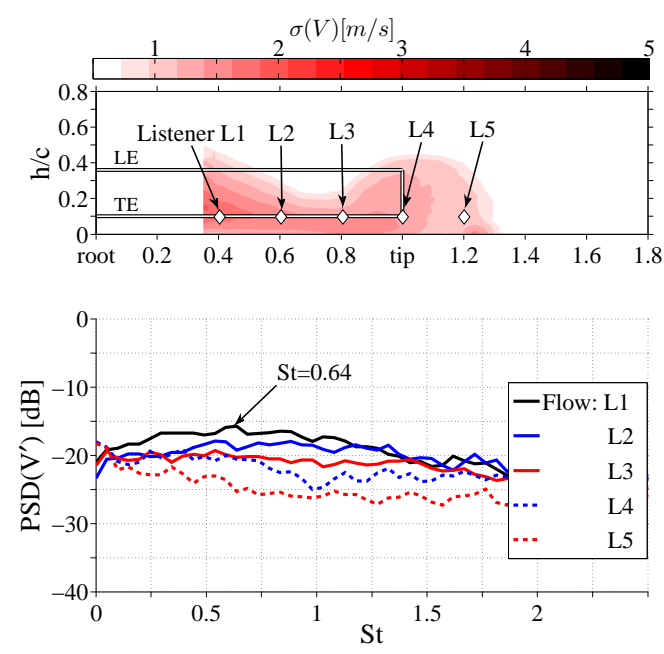

(e) Rigid flat-plate, $\mathrm{h} / \mathrm{c}=0.1, \alpha=15^{\circ}$
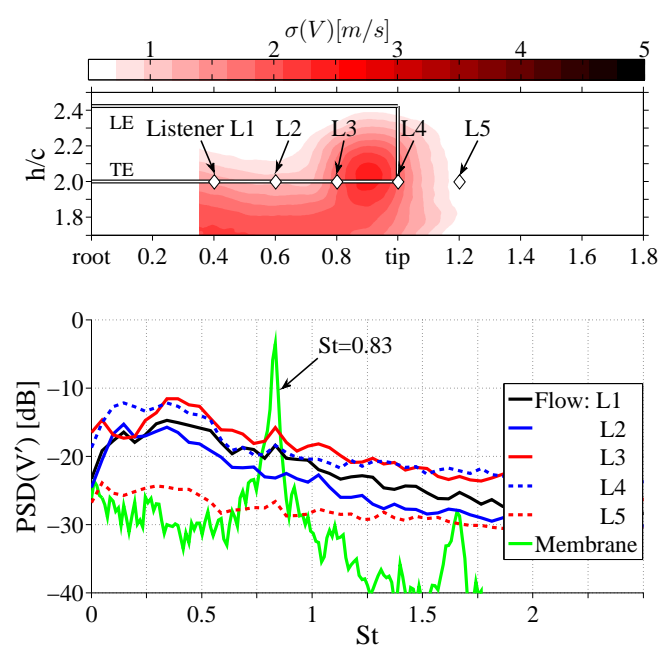

(b) Membrane, $\mathrm{h} / \mathrm{c}=2, \alpha=25^{\circ}$
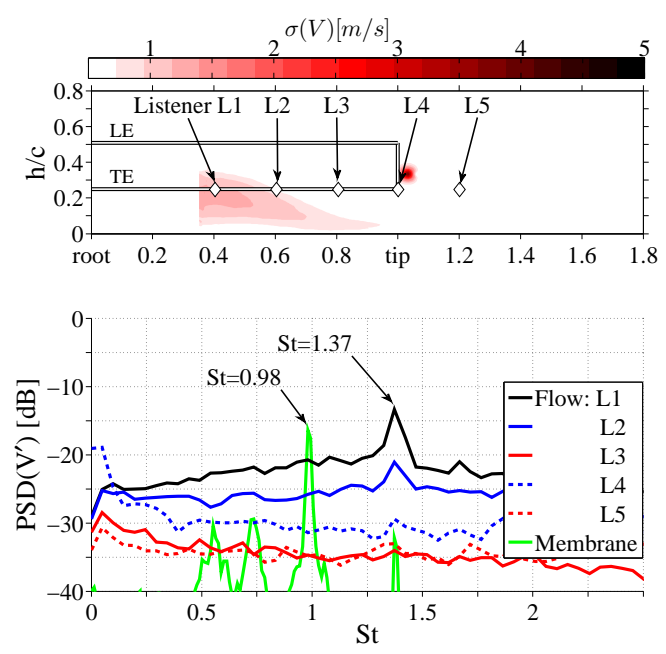

(d) Membrane, $\mathrm{h} / \mathrm{c}=0.25, \alpha=15^{\circ}$
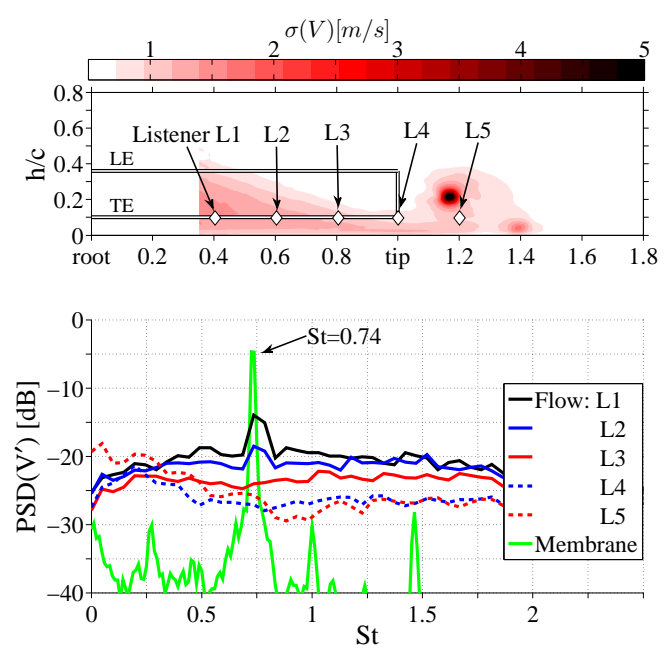

(f) Membrane, $\mathrm{h} / \mathrm{c}=0.1, \alpha=15^{\circ}$

Fig. 14 PSD of flow and membrane dynamics, comparing flat-plate and membrane wings. 
Fig. 15 gives an extended overview into the coupling of aerodynamic loads, membrane oscillations and flow dynamics. We focus on the highly coupled case at $\alpha=15^{\circ}$ and $h / c=0.1$ for brevity. Fig. 15a shows the timeseries, Fig. 15b the spectral content and Fig. 15c the cross-correlation between load, membrane and flow dynamics. Membrane fluctuations are shown at the surface point with maximum membrane standard-deviation (STD), located at $40 \%$-chord from the leading-edge in mid-plane. Vertical velocity fluctuation in the wake are shown at the listener point $L 1$, located $0.4 \mathrm{c}$ spanwise from the wing root at a height of $h / c=0.1$. The time-series in Fig. 15a reflects predominant frequencies in load, membrane and flow dynamics. The membrane signal has the lowest background-noise level in comparison to load and flow dynamics. The spectra in Fig. 15b reveals that the dominant membrane eigen frequencies can directly influence the flow and load content, which are known to enhance the wings stall-performance through unsteady flow reattachment (Bleischwitz et al., 2017).

The cross-correlation between the flow and the membrane (Fig. 15c, upper), the loads and the membrane (Fig. 15c, mid) and the loads and the flow (Fig. 15c, lower) underlines the coupling strength and phase relation between each other. The aerodynamic coefficients of lift, drag and pitch show strong correlation to the membrane dynamics

(Fig. 15c, mid). Local upward deflection of the membrane near the leading-edge correlates with a nose-up pitching moment, reduced lift and increased drag. This result is consistent with our previous findings (Bleischwitz et al., 2017).

The correlation between the vertical velocity in the wake of the wing and membrane oscillations is found to be 0.45. This correlation value appears to be reduced because the flow exhibits a broader range of scales in comparison to the distinct membrane oscillation signal (see also Fig. 15a). The flow appears to have a phase-lag of $-30^{\circ}$ with the membrane deformation. The correlation values between the loads and the flow (Fig. 15c, lower) are found to be about 0.3. This lower value could be due to two reasons. First, as mentioned before, the flow has a large range of scales, and second, the load-cell signal does include the influence of other noise sources as well as there are limits to its resolution (see Fig. 4.

Given that the dominant frequency in the wake, the forces/moment and the membrane fluctuations all exhibit a similar behaviour, it is hypothesised that the membrane is primarily responsible for setting this frequency. However, the extent to which the membrane oscillations can alter flow and load dynamics could entirely depend on the fundamental membrane frequency, vibration amplitude and the type of oscillation. Further studies with actively controlled membrane dynamics might help to reveal such limitations.

\subsection{Flow-Structure Coupling}

Fig. 16 summarises the fluid-structure coupling by showing cycle-averaged load (column Fig. 16a), membrane (column Fig. 16b) and wake-flow dynamics (column Fig. 16c), illustrated for the highly coupled membrane wing case at $\alpha=15^{\circ}$ in extreme ground-effect conditions $(h / c=0.1)$. The cycle-average comprises of over 370 individual (membrane) cycles with a dominant frequency of $\mathrm{St}=0.74$, ranging over a total (DIC + PIV) recording period of $6.25 \mathrm{~s}$. The resulting membrane cycle measures 13 phase points per complete $360^{\circ}$-cycle. The cycle averaged loads show lift, drag and pitch moment fluctuations which are normalised such that their peak value is 1 (Fig. 16a). Flow fluctuations (Fig. 16c) are first low-pass filtered using the first five POD-modes (red = upwards, blue = downwards velocity) and then cycle-averaged over one membrane cycle. Vortical flow structures are shown using the Q-criterion and extruded (using frozen flowfield hypothesis) with increasing phase-cycle from the initial PIV measurement plane. For clarity, flow structures are mirrored about the root (assuming symmetry). A listener-point for vertical flow fluctuations $\left(\left|V_{L 1}^{\prime}\right|\right)$ is placed within the PIV-plane $0.4 \mathrm{c}(40 \mathrm{~mm})$ from the wing center plane and $0.15 \mathrm{c}$ from the ground $(15 \mathrm{~mm})$ and allows to compare the dynamics of vortex-shedding with membrane (Fig. 16b) and load-dynamics (Fig. 16a). Vertical upward fluctuations of the membrane wing dynamics are amplified for clarity by factor 7 and are color-coded in red for upwards and blue for downwards motions (consistent to up-/ and downwards color coding of the flow fluctuations).

The vertically illustrated phase-cycle of Fig. 16 shows at the beginning of the cycle at $0^{\circ} \leq$ Phase $\leq 60^{\circ}$ an instantaneous upwards membrane motion (red) close to the leading-edge (LE) which correlates well with an upward (red) shedding related flow fluctuation at listener point $L 1$. This vertical velocity fluctuation relates to a previous cycle which convected spatially with the flow. Low lift, high drag and a pitch-up (nose-up) moment correlate to this membrane deformation. At a period of Phase $\geq 180^{\circ}$, the downwards facing membrane motion close to the LE correlates to downwards facing flow features (blue) in the wake-plane, resulting in increased lift, reduced drag and a growing pitch-down moment. The results reveal the strong spatial and temporal link between the dynamics of the wing structure and their impact on load dynamics and correlating flow feature dynamics in the wake. For further visualisation and clarity, Fig. 16 is available as supplementary video-file Video-Fig.16. 

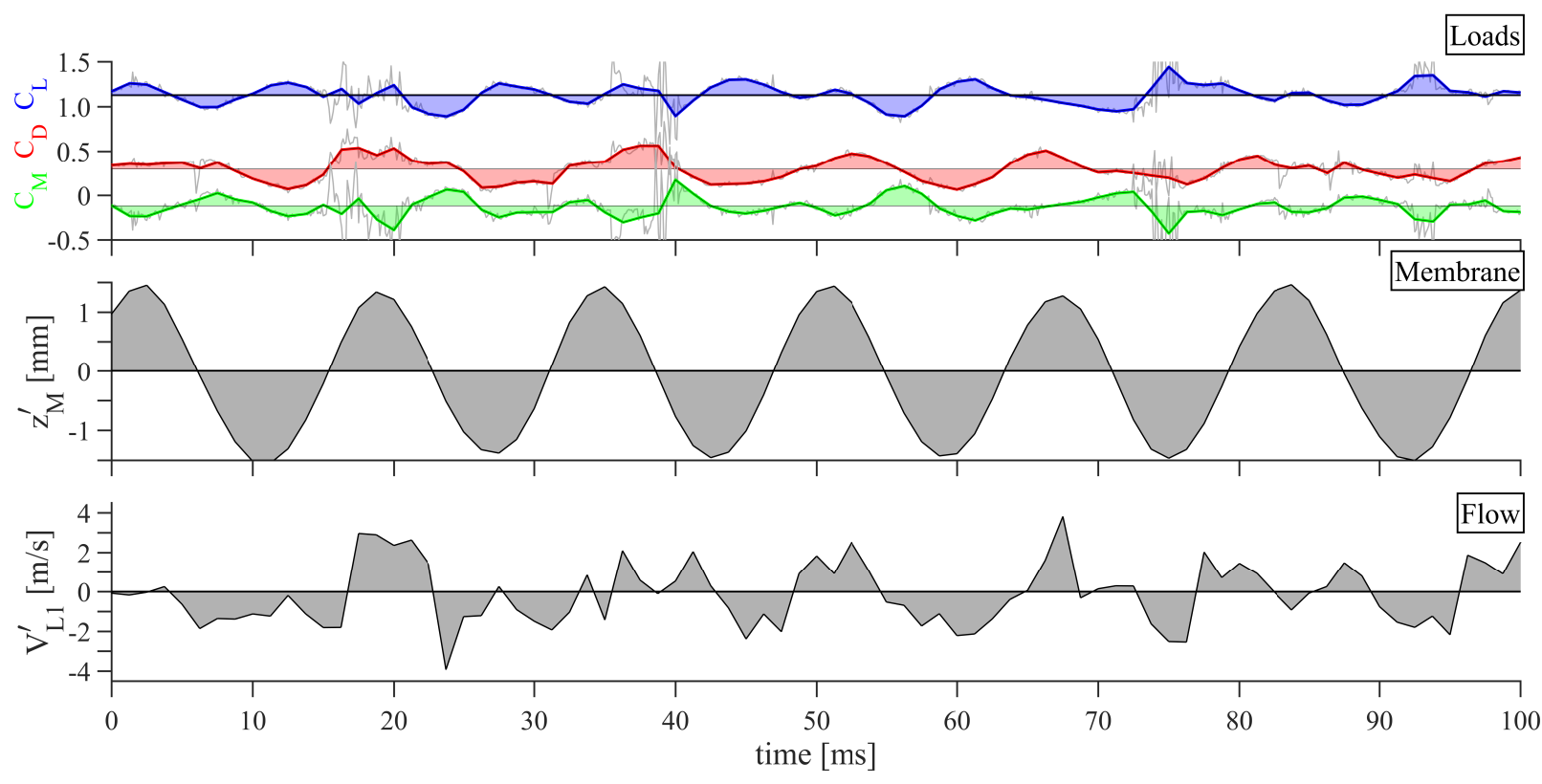

(a) Timeseries
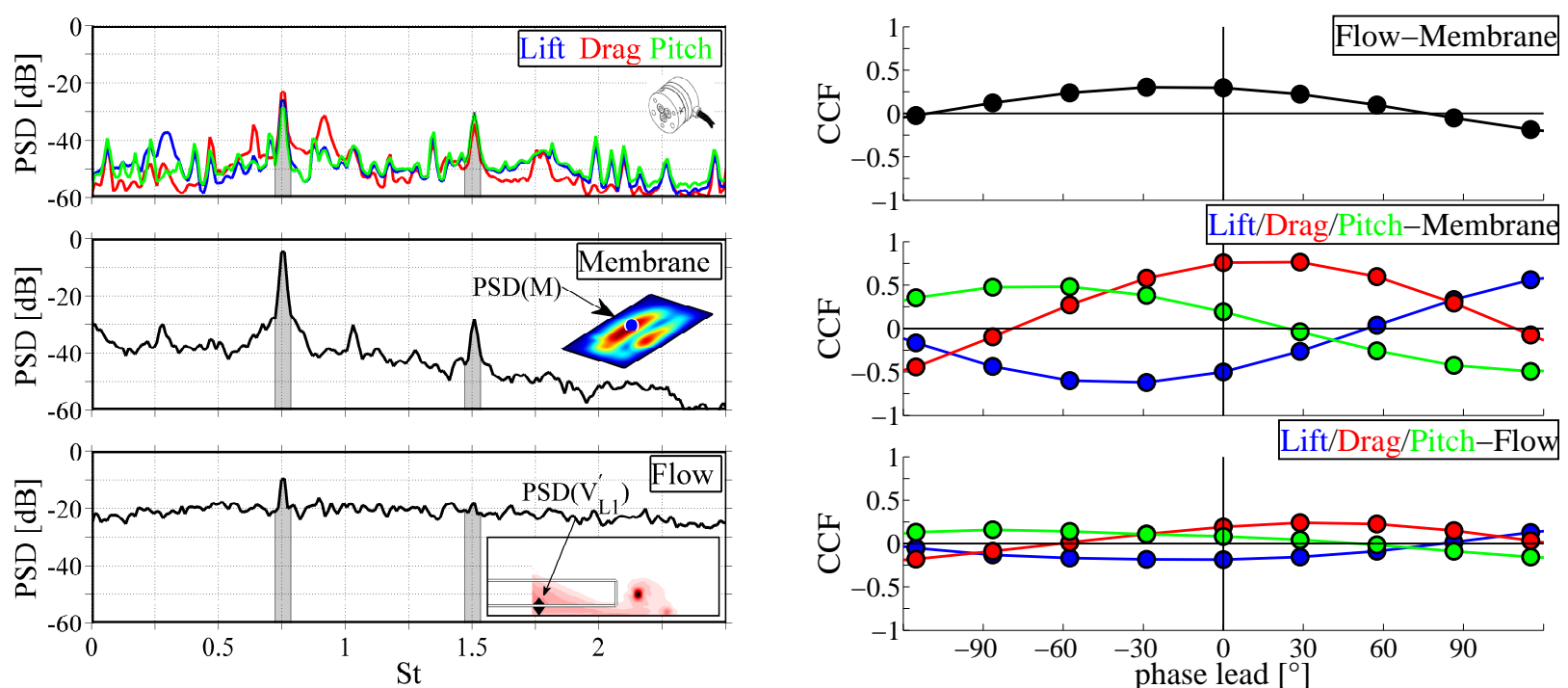

(b) Spectra

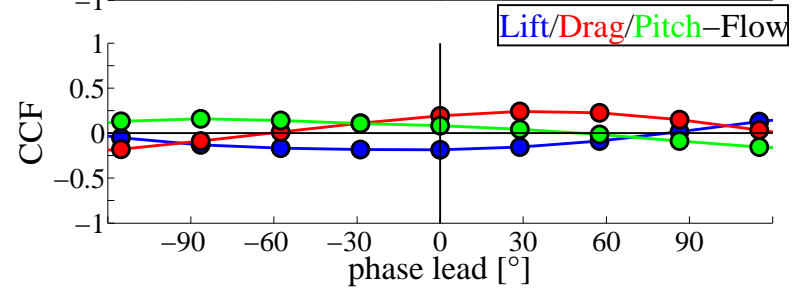

(c) Cross-correlation

Fig. 15 Timeseries (a), spectra (b) and phase (c) relation between aerodynamic loads, membrane fluctuations (at peak STD, 40\%chord from LE) and flow (at listener Ll) fluctuations. Exemplary highly coupled case at $\alpha=15^{\circ}$ in extreme ground-effect $(h / c=$ $0.1)$. 

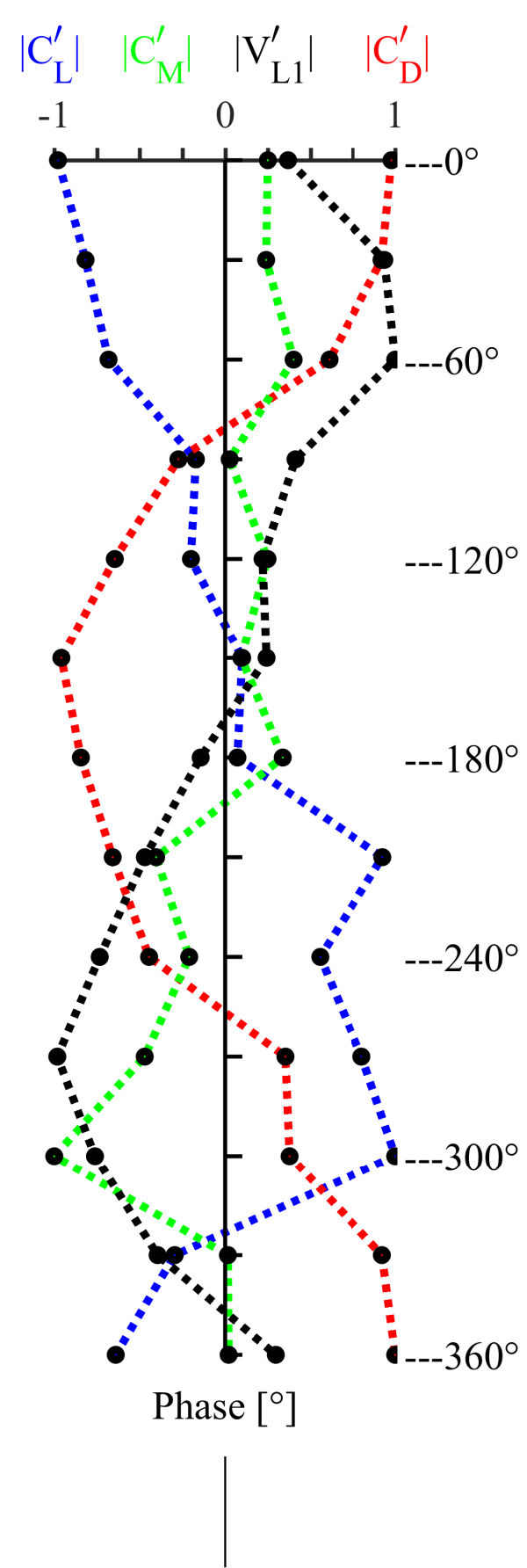

(a)

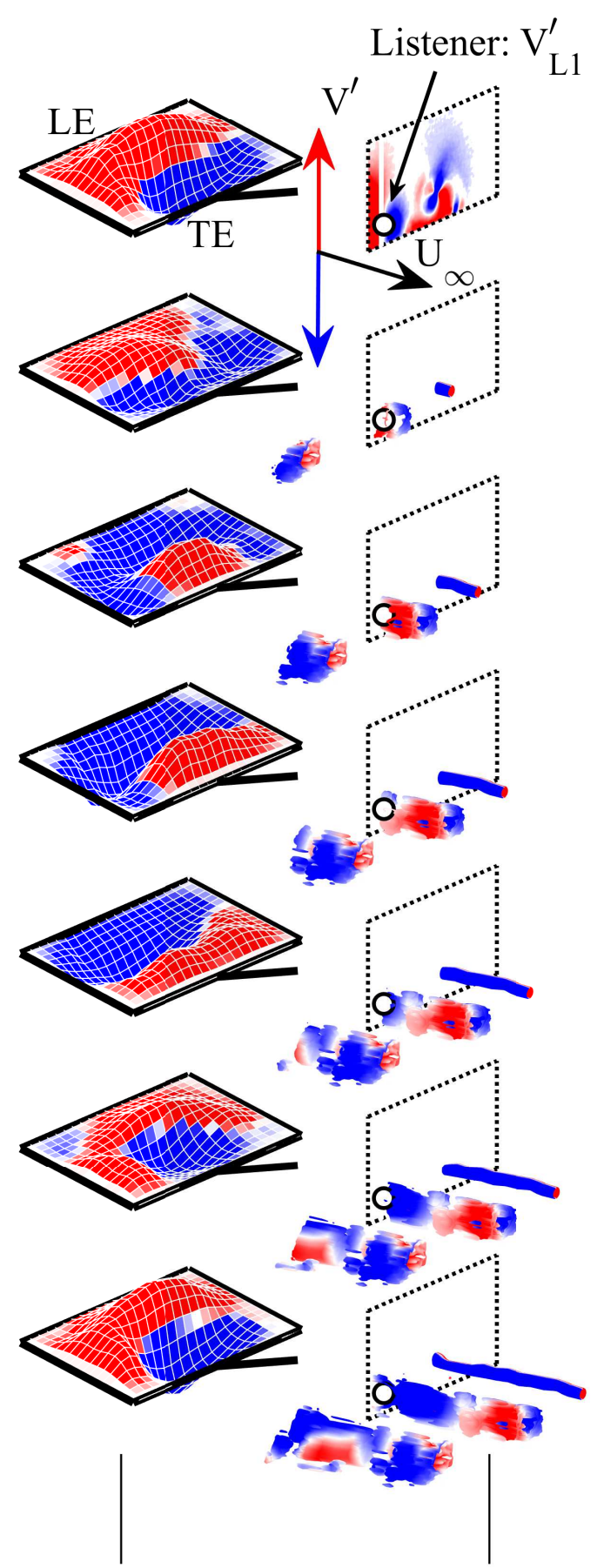

(b)

(c)

Fig. 16 Vertical phase-cycle of load-membrane-flow fluctuations, ranging from $0^{\circ} \leq$ Phase $\leq 360^{\circ}$.

(a) Normalised load-coefficients $C_{L}^{\prime}, C_{D}^{\prime}, C_{M}^{\prime}$ and vertical flow fluctuations $V_{L 1}^{\prime}$ at listener-point L1.

(b) Corresponding membrane surface fluctuations at seven phase locations (without mean, red $=$ UP).

(c) Q-criteria related wake flow structures, extruded with envolving phase-angle out of the PIV-plane.

Case: Highly coupled membrane wing at $\alpha=15^{\circ}$ and $h / c=0.1$.

For illustration of the video Video-Fig.16, the reader is referred to the web version of this paper. 


\section{Conclusion}

Wind tunnel experiments were conducted at moderate Reynolds numbers of $\operatorname{Re}=56,000$, investigating tip-vortex modifications of rectangular rigid flat-plate and membrane wings at a freestream-normal plane, located one chord downstream of the wings trailing-edge. Both wings are measured from free-flight into ground-effect conditions. Timesynchronised high-speed recordings of aerodynamic loads, membrane motions and flow dynamics in the wake reveal underlying fluid-structure interaction of flexible membrane wings.

For attached flow conditions, ground-effect is found to benefit both rigid and membrane wings in their lifting and aerodynamic performance. Their benefits relate both on an increase in static pressure on the lower side of the wing and a spanwise push-out effect of the tip-vortices close to the ground, resulting in an enlarged virtual aspect-ratio with low induced drag content.

However, for unsteady flow conditions, initiated by very high angles-of-attack or deep ground-effect conditions, membrane wings are able to adapt their static shape by passive cambering and allow membrane oscillations to couple with vortical-shedding structures from the leading-edge. As a result, membrane wings are found to dynamically reattach the flow above the wing upper surface, stabilize the tip-vortices in unsteady flow conditions and ultimately delay the tip-vortex break-down for an enhanced performance window. While exposed to ground-effect conditions, membrane wings show in comparison to rigid flat-plates a performance gaining $20 \%$ higher tip-vortex push-out distance.

Nevertheless, this pretended aerodynamic performance gain of membrane wings in ground-effect appears only valid within certain (coupling-enabled) unsteady flow conditions and gains are often counterbalanced by an increase in drag due to cambering related, additional lift generation of the membrane wing.

From a certain high level of flow unsteadiness, caused by extreme ground-effect and/or high angles-of-attack, static cambering and dynamic oscillations of the membrane is not anymore sufficient to reach lag-free fluid-membrane coupling conditions. From this point, rigid flat-plate and membrane wings start to behave aerodynamically similar and the wing performance appears rather dominated from the lower than the upper-side of the wing.

Importantly, both the current wake-oriented and prior streamwise studies reveal that the eigen frequencies of welldefined passive membrane wings can alter and couple with dominant shedding frequencies in the flow, which appear different to typical (pure geometry-related) shedding frequencies of rigid flat/cambered plates. The results reveal the potential of flexible membrane wings to exploit and even drive fluid-structure coupling in separated flow conditions. In addition, a path is laid for a new type of Micro-Air-Vehicles, combining flow adapting flexible membrane wings in aerodynamic performance enlarging ground-effect conditions.

\section{Further work}

Coupling between the flow and a passive adaptive membrane wing can only occur for a small selection of resonating membrane-to-flow conditions. The selection of the membrane material, such as the membrane thickness, material stiffness, pre-tension, roughness, attachment method or the wing planform shape fixes the resulting coupling conditions. Electro-mechanic or electro-active membrane wing systems could allow to customise and find beneficial membrane motions on demand, as they could allow modifications in membrane oscillation frequencies, changes in activation patterns or scalable oscillation amplitudes. Mechanical systems could include a system by which the membrane can be rolled around the trailing-edge for static camber changes and swivelled around the leading-edge to promote dynamic membrane oscillations. Ultimately, due to dynamic interactions of the membrane with the flow, the key relies on the ability to manipulate the membrane/flow oscillations in such a way that positive lift and negative drag fluctuations are actively encouraged and negative lift and positive drag fluctuations are actively suppressed.

\section{Acknowledgements}

We gratefully acknowledge the support from the Engineering and Physical Sciences Research Council (EPSRC, grant EP/J001465/1) and the European Office of Aerospace Research and Development (EOARD, grant FA865512-1-2046). Dr. R. de Kat was partially supported by a Leverhulme Trust Early Career Fellowship. In addition, we gratefully acknowledge LaVision, and in specific Dr. A. Nila for their/his individual and always timely support. Figure related data in this paper is available from the University of Southampton repository at: http://dx. doi .org/10. 5258/SOTON/XXXXXX. 


\section{References}

Ahmed, M., Sharma, S., jul 2005. An investigation on the aerodynamics of a symmetrical airfoil in ground effect. Experimental Thermal and Fluid Science 29 (6), 633-647.

https://doi.org/10.1016/j.expthermflusci.2004.09.001

Ahmed, M. R., Takasaki, T., Kohama, Y., Lecturer, S., Campus, L., Turbulence, L., Tunnel, W., 2006. Experiments on the Aerodynamics of a Cambered Airfoil in Ground Effect. In: 44th AIAA Aerospace Sciences Meeting and Exhibit. No. January. Reno, Nevada. http://dx.doi.org/10.2514/6.2006-258

Arce, M., Ukeiley, L., Hubner, J., Ifju, P., 2013. Passively Compliant Membranes in Low Aspect Ratio Wings. In: 51st AIAA Aerospace Sciences Meeting including the New Horizons Forum and Aerospace Exposition. Grapevine (Dallas/Ft. Worth Region), Texas. https://doi.org/10.2514/6.2013-681

Bleischwitz, R., 2016. Fluid-structure interactions of membrane wings in free-flight and in ground-effect. Phd-thesis, University of Southampton. https : //eprints. soton.ac.uk/397261/

Bleischwitz, R., de Kat, R., Ganapathisubramani, B., 2015a. Aeromechanics of Membrane Wings in Ground-Effect. In: 45th AIAA Fluid Dynamics Conference. Dallas, Texas, pp. 1-28. http://dx.doi.org/10.2514/6.2015-2764

Bleischwitz, R., de Kat, R., Ganapathisubramani, B., 2015b. Aspect-Ratio Effects on Aeromechanics of Membrane Wings at Moderate Reynolds Numbers. AIAA Journal 53 (3), 780-788. http://dx.doi.org/10.2514/1.J053522

Bleischwitz, R., de Kat, R., Ganapathisubramani, B., 2017. On the fluid-structure interaction of flexible membrane wings for MAVs in and out of ground-effect. Journal of Fluids and Structures 70, 214-234. https://doi.org/10.1016/j.jfluidstructs.2016.12.001

Bleischwitz, R., Kat, R. D., Ganapathisubramani, B., 2016. Aeromechanics of membrane and rigid wings in and out of ground-effect at moderate Reynolds numbers. Journal of Fluids and Structures 62, 318-331. http://dx.doi.org/10.1016/j.jfluidstructs.2016.02.005

Dubief, Y., Delcayre, F., 2000. On coherent-vortex identification in turbulence. Journal of Turbulence 1 (11), 1-22. http://dx.doi.org/10.1088/1468-5248/1/1/011

Galvao, R., Israeli, E., Song, A., Tian, X., Bishop, K., Swartz, S., Breuer, K. S., 2006. The Aerodynamics of Compliant Membrane Wings Modelled on Mammalian Flight Mechanics. In: 36th AIAA Fluid Dynamics Conference and Exhibit. San Francisco, California. https://doi.org/10.2514/6.2006-2866

Gordnier, R. E., 2009. High fidelity computational simulation of a membrane wing airfoil. Journal of Fluids and Structures $25,897-917$. https://dx.doi.org/10.1016/j.jfluidstructs.2009.03.004

Gordnier, R. E., Attar, P. J., 2014. Impact of flexibility on the aerodynamics of an aspect ratio two membrane wing. Journal of Fluids and Structures $45,138-152$.

https://dx.doi.org/10.1016/j.jfluidstructs.2013.10.004

Han, C., Cho, J., 2005. Unsteady Trailing Vortex Evolution Behind a Wing in Ground Effect. Journal of Aircraft 42 (2), $429-434$. https://dx.doi.org/10.2514/1.6477

Lumley, J. L., 1967. The structure of inhomogeneous turbulent fows. Atmospheric turbulence and radio wave propagation, 166-178. http://dx.doi.org/10.1016/S0376-0421(03)00076-9

Miranda, S., Vlachos, P. P., Telionis, D. P., Corporation, A., 2005. Flow Control of a Sharp-Edged Airfoil. AIAA Journal 43 (4), $716-726$. http://dx.doi.org/10.2514/1.2725

Park, S. G., Kim, B., Sung, H. J., 2017. Hydrodynamics of a self-propelled flexible fin near the ground. Physics of Fluids 29 (5), 051902. http://dx.doi.org/10.1063/1.4983723

Pinnau, R., 2008. Model Reduction via Proper Orthogonal Decomposition. In: Model Order Reduction: Theory, Research Aspects and Applications. Springer Berlin Heidelberg, pp. 95-109.

http://dx.doi.org/10.1007/978-3-540-78841-6\{_\}5

Quinn, D. B., Lauder, G. V., Smits, A. J., 2014. Flexible propulsors in ground effect. Bioinspiration \& Biomimetics $9,036008$. https://doi.org/10.1088/1748-3182/9/3/036008

Rojratsirikul, P., Genc, M. S., Wang, Z., Gursul, I., 2011. Flow-induced vibrations of low aspect ratio rectangular membrane wings. Journal of Fluids and Structures 27, 1296-1309.

http://dx.doi.org/10.1016/j.jfluidstructs.2011.06.007

Rojratsirikul, P., Wang, Z., Gursul, I., 2010a. Effect of pre-strain and excess length on unsteady fluid-structure interactions of membrane airfoils. Journal of Fluids and Structures 26, 359-376.

http://dx.doi.org/10.1016/j.jfluidstructs.2010.01.005

Rojratsirikul, P., Wang, Z., Gursul, I., 2010b. Unsteady Aerodynamics of Low Aspect Ratio Membrane Wings. In: 48th AIAA Aerospace Sciences Meeting. No. January. Orlando,Florida. http://dx.doi.org/10.2514/6.2010-729

Schreier, H. W., Braasch, J. R., Sutton, M. A., 2000. Systematic Errors in Digital Image Correlation caused by Intensity Interpolation. Optical Engineering 39 (November 2000), 2915-2921. http://dx.doi.org/10.1117/1.1314593

Sirovich, L., 1987. Turbulence and the dynamics of coherent structures, I-III. Quarterly of Apploed Mathematics 45 (3), 561-590. http://tinyurl.com/Sirovich1987

Smith, R., Shyy, W., 1996. Computation of aerodynamic coefficients for a flexible membrane airfoil in turbulent flow: A comparison with classical theory. Physics of Fluids 8 (12), 3346-3353. http://dx.doi.org/10.1063/1.869122 
Song, A., Tian, X., Israeli, E., Galvao, R., Bishop, K., Swartz, S., Breuer, K., 2008. Aeromechanics of Membrane Wings with Implications for Animal Flight. AIAA Journal 46 (8), 2096-2106.

http://dx.doi.org/10.2514/1.36694

Stanford, B., Ifju, P., Albertani, R., 2014. Fixed Membrane Wings for Micro Air Vehicles : Experimental Characterization , Numerical Modeling , and Tailoring. Bret Kennedy Stanford, 1-53.

https://doi.org/10.1016/j.paerosci.2008.03.001

Timpe, A., Zhang, Z., Hubner, J., Ukeiley, L., 2013. Passive flow control by membrane wings for aerodynamic benefit. Experiments in Fluids $54(1471), 1-23$ http://dx.doi.org/10.1007/s00348-013-1471-0

Vogt, J., Barber, T., 2012. Ground effect phenomena about lift and downforce generating cambered aerofoils. International Journal of Numerical Methods for Heat \& Fluid Flow 22 (2), 153-174

http://dx.doi.org/10.1108/09615531211199809

Yun, L., Bliault, A., Doo, J., 2010. WIG Craft and Ekranoplan: Ground Effect Craft Technology. In: Springer, ISBN: 9781441900425.

http://dx.doi.org/10.1007/978-1-4419-0042-5

Zhang, Z., Martin, N., Wrist, A., Hubner, J. P., 2016. Geometry and Prestrain Effects on the Aerodynamic Characteristics of Batten-Reinforced Membrane Wings. Journal of Aircraft 53 (2), 1-15. http://dx.doi.org/10.2514/1.C033386

Zhang, Z., Wrist, A., Hubner, J. P., 2017. Effects of leading-edge vibration on a spanwise-tensioned membrane at low Reynolds number. Journal of Fluids and Structures 69, 441-457.

https://doi.org/10.1016/j.jfluidstructs.2016.12.009 